\Genome Biology

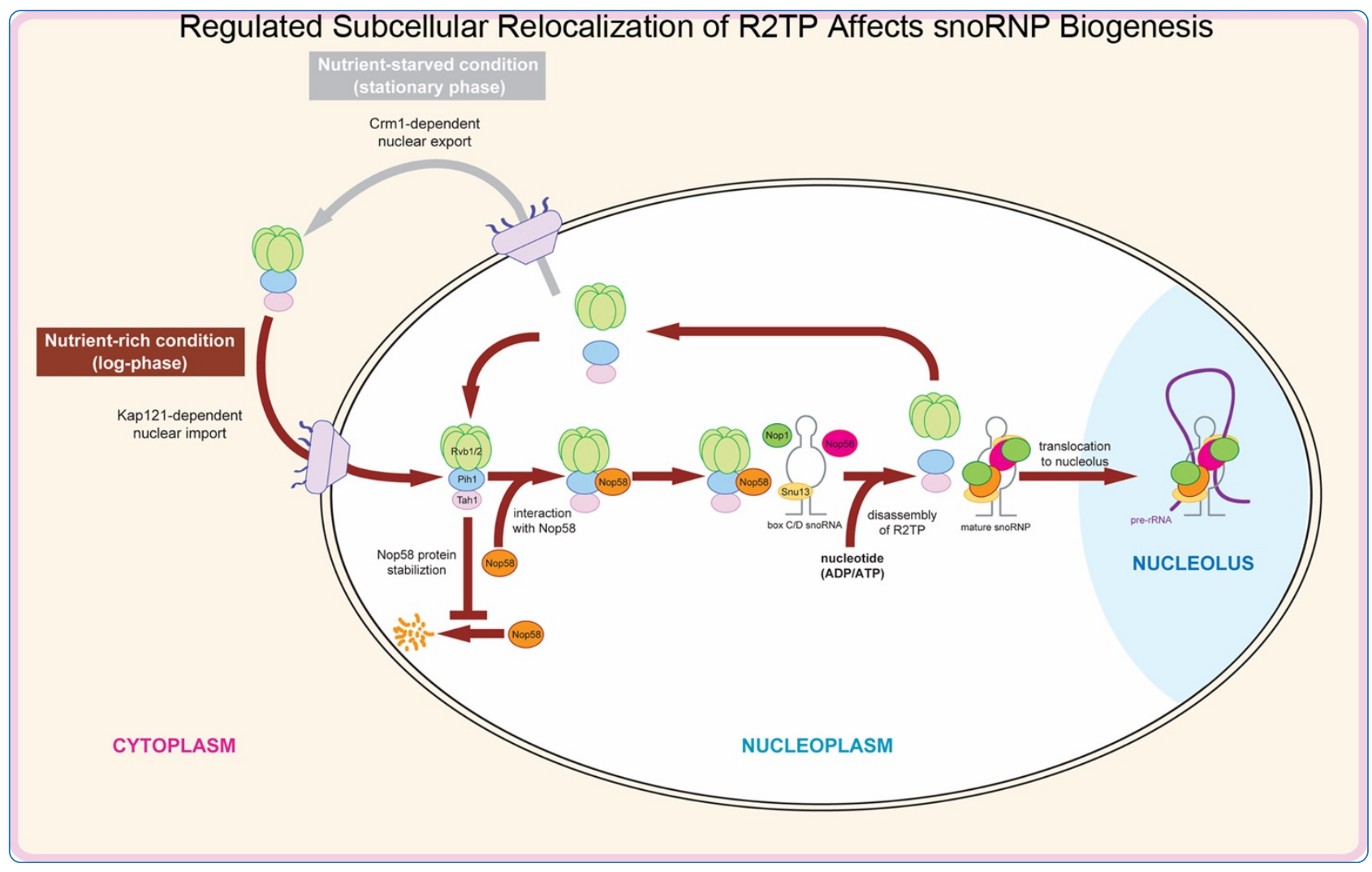

\title{
Nutritional status modulates box C/D snoRNP biogenesis by regulated subcellular relocalization of the R2TP complex
}

Kakihara et al. 


\title{
Nutritional status modulates box C/D snoRNP biogenesis by regulated subcellular relocalization of the R2TP complex
}

\author{
Yoshito Kakihara, Taras Makhnevych, Liang Zhao, Weiwen Tang and Walid A Houry*
}

\begin{abstract}
Background: Box C/D snoRNPs, which are typically composed of box C/D snoRNA and the four core protein components Nop1, Nop56, Nop58, and Snu13, play an essential role in the modification and processing of pre-ribosomal RNA. The highly conserved R2TP complex, comprising the proteins Rvb1, Rvb2, Tah1, and Pih1, has been shown to be required for box C/D snoRNP biogenesis and assembly; however, the molecular basis of R2TP chaperone-like activity is not yet known.
\end{abstract}

Results: Here, we describe an unexpected finding in which the activity of the R2TP complex is required for Nop58 protein stability and is controlled by the dynamic subcellular redistribution of the complex in response to growth conditions and nutrient availability. In growing cells, the complex localizes to the nucleus and interacts with box C/D snoRNPs. This interaction is significantly reduced in poorly growing cells as R2TP predominantly relocalizes to the cytoplasm. The R2TP-snoRNP interaction is mainly mediated by Pih1.

Conclusions: The R2TP complex exerts a novel regulation on box C/D snoRNP biogenesis that affects their assembly and consequently pre-rRNA maturation in response to different growth conditions.

\section{Background}

The ribosome is a highly complex machinery dedicated to protein synthesis. Its biogenesis involves a large number of protein and RNA factors consuming a considerable portion of the cellular energy [1,2]. Eukaryotic cells require more than 70 ribosomal proteins (r-proteins) and four distinct rRNAs (25S/28S, $18 \mathrm{~S}, 5.8 \mathrm{~S}$, and $5 \mathrm{~S})$ for ribosome formation. In yeast, three of the four rRNAs $(25 \mathrm{~S}, 18 \mathrm{~S}$, and $5.8 \mathrm{~S})$ are generated from a $35 \mathrm{~S}$ pre-rRNA, which has to be extensively processed and modified into the mature rRNAs. The processing of pre-rRNA is carried out by a large number of small nucleolar ribonucleoproteins (snoRNPs) and numerous non-ribosomal trans-acting factors such as endo- and exonucleases and RNA helicases [1,3-5].

There are two major classes of snoRNPs, box C/D and box $\mathrm{H} / \mathrm{ACA}$, that are responsible for 2'-O-methylation and pseudouridylation of pre-rRNA, respectively $[1,6]$. The box C/D snoRNPs consist of box C/D snoRNAs

\footnotetext{
* Correspondence: walid.houry@utoronto.ca

1 King's College Circle, Medical Sciences Building, Department of

Biochemistry, University of Toronto, Toronto, Ontario M5S 1A8, Canada
}

and four core proteins: Snu13, Nop1, Nop56, and Nop58. The snoRNA guides the snoRNP complex to the methylation site on the pre-rRNA by base pairing $[7,8]$. Nop1 (fibrillarin in vertebrates), which contains the signature motif of S-adenosyl-methionine RNA methyltransferase, catalyzes the methylation of the 2 '-hydroxyl group of the target ribose on the pre-rRNA $[9,10]$.

It has been proposed that box C/D snoRNPs assemble hierarchically. In vertebrates, the formation of box $\mathrm{C} / \mathrm{D}$ snoRNA-15.5 K (Snu13 ortholog) complex is required for the assembly of the other core proteins with the snoRNP [11]. Similarly, stepwise association has been observed in archaea beginning with the interaction between box C/D sRNA and L7Ae (Snu13 homolog), followed by the binding of Nop5 (Nop56/Nop58 homolog) and then the recruitment of fibrillarin into the complex [10]. A number of protein factors are required for the assembly of snoRNPs. These factors include the R2TP complex, Hsp90, Bcd1, Rsa1, and Srp40/Nopp140 in yeast [12-15]. Other additional factors in higher eukaryotes include TAF9, PHAX, CRM1, CBC, Ran, and Snurportin1 [16-20]. 
The R2TP complex was discovered by our group in yeast S. cerevisiae [21]; it is composed of four proteins: Rvb1, Rvb2, Tah1, and Pih1. The complex is highly conserved from yeast to mammals [22]. Rvb1 and Rvb2 are members of AAA + (ATPase associated with diverse cellular activities) superfamily and, in addition to snoRNP assembly, are involved in many other critical cellular processes such as chromatin remodeling, DNA replication, DNA damage repair, transcription, and telomerase assembly [23,24]. Pih1 (also known as Nop17) was initially isolated as a Nop58 interacting protein in yeast [25] and, subsequently, shown to be part of the R2TP complex and to interact with Hsp90 [14,21]. Tah1 contains two tetratricopeptide repeat (TPR) motifs and acts as a co-factor for Hsp90 to stabilize Pih1 [14,26]. We and others have shown that the R2TP complex functions as a snoRNP assembly factor in yeast [14] and mammalian cells [13]. More recently, the complex has been found to be also required for other cellular processes in mammals such as assembly of RNA polymerase II $[27,28]$, phosphatidylinositol 3-kinase-related kinases (PIKKs) signaling pathway [29], and apoptosis [30-32].

Here, we show that the R2TP complex strongly interacts with unassembled Nop58, that the function of R2TP in box C/D snoRNP biogenesis is mediated by the interaction of Pih1 with Nop58, and that the R2TP complex stabilizes Nop58. Importantly, the R2TP-Nop58 interaction was found to be growth-phase or nutrientcondition dependent. Also, we uncovered a shift in the cellular distribution of R2TP proteins between the cytoplasm and the nucleus based on the growth phase of the cell and nutrient condition. Our data suggest that this dynamic subcellular relocation of the R2TP complex could regulate its function, which, subsequently, affects box C/D snoRNP assembly and, hence, modulates prerRNA processing. Our findings describe a novel regulatory mechanism for box $\mathrm{C} / \mathrm{D}$ snoRNP biogenesis in response to different growth conditions.

\section{Results}

\section{R2TP interacts with Nop58 through Pih1}

We previously identified genetic interactions between RVB1, RVB2, and PIH1 with NOP58, and showed that the R2TP complex is required for box C/D snoRNA accumulation and box C/D snoRNP assembly [14]. Also, Gonzales et al. [25] showed a physical interaction between Pih1 and Nop58. However, the detailed molecular basis of R2TP complex function in box C/D snoRNP biogenesis remains unknown. To investigate whether R2TP proteins physically interact with box C/D snoRNP complexes, we constructed C-terminal FLAG-tagged Snu13, Nop1, Nop56, and Nop58 strains, and performed pulldown assays using cell lysate extracted from log phase cells followed by Western blot analysis (Figure 1A).
All the R2TP proteins were found to significantly interact with Nop58, while Nop1, Snu13, and Nop56 exhibit weak interaction with Rvb1/2 only (Figure 1A). Subsequently, we treated the soluble cell lysate extracted from the Nop58-FLAG strain with RNase A prior to the pulldown in order to disassemble the snoRNPs by degrading the associated snoRNAs. The lysate was also treated with DNase I to degrade DNA as a control (Figure 1B). RNase A but not DNase I treatment reduced the levels of Snu13, Nop1, and Nop56 bound to Nop58-FLAG indicating that the snoRNPs were partially disassembled as expected, whereas no such dissociation was observed for snoRNPs isolated from DNase I-treated cell lysate. Intriguingly, RNase A treatment resulted in increased interaction of R2TP with Nop58-FLAG compared to no treatment or to DNase I-treated sample. These results suggest that the R2TP complex predominantly interacts with Nop58 that is likely released from box C/D snoRNP complexes and that snoRNAs are not required for the R2TP-Nop58 interaction.

In order to determine which protein component of the R2TP complex mediates the interaction with Nop58, we co-immunopurified Nop58-FLAG complex from WT, pih1s, and tah1s log phase cells. The levels of Rvb1, Rvb2, Pih1, Tah1, Snu13, Nop1, and Nop56 bound to Nop58-FLAG were determined by Western blot analysis or silver staining. As shown in Figure $1 C$, the deletion of PIH1 but not of TAH1 significantly reduced the interaction of Rvb1/2 with Nop58. Furthermore, the binding of Tah1 to Nop58-FLAG required Pih1 (Figure 1C). The decreased levels of Nop1, Nop56, and Snu13 interacting with Nop58-FLAG in pih1 $\Delta$ cells (Figure 1C) indicates that the snoRNP complex is not properly assembled in these cells. This is consistent with our previous observations in which we noted that the levels of Nop1 and Snu13 that co-purified with Nop56-FLAG were decreased in pih1s strain [14]. This result indicates that Pih1 enhances the interaction between R2TP and Nop58.

To determine the binding site of Pih1 on Nop58 (511 amino acids), we generated N-terminal GST fusion constructs of different domains of Nop58 based on the available crystal structures of the equivalent archaeal protein $[33,34]$ and expressed them in E. coli. Nop58 can be divided into three functional domains (Figure 1D, top panel): N-terminal domain that binds Nop1, middle domain that includes a coiled-coil motif which mediates self-dimerization, and a C-terminal domain, also known as the Nop domain, that interacts with both L7Ae (Snu13 in yeast) and the box C/D sRNA in archaea. The Cterminal domain is followed by a KKE/D repeat (Figure 1D, top panel). GST-fused full-length Nop58 did not express well in $E$. coli (data not shown); but a construct deleted of the C-terminal KKE/D repeat (Figure 1D, top panel), GST-Nop58-447, expressed well and was purified. 
B

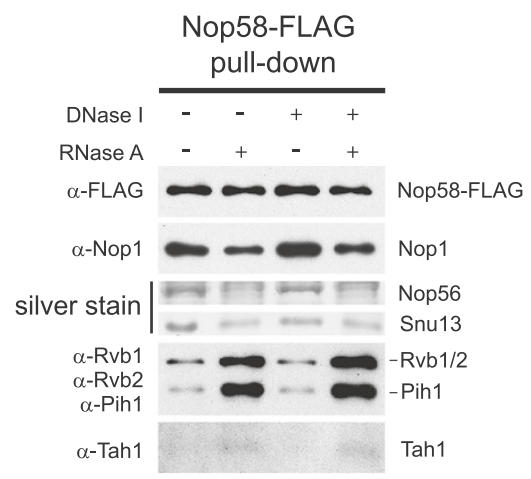

D
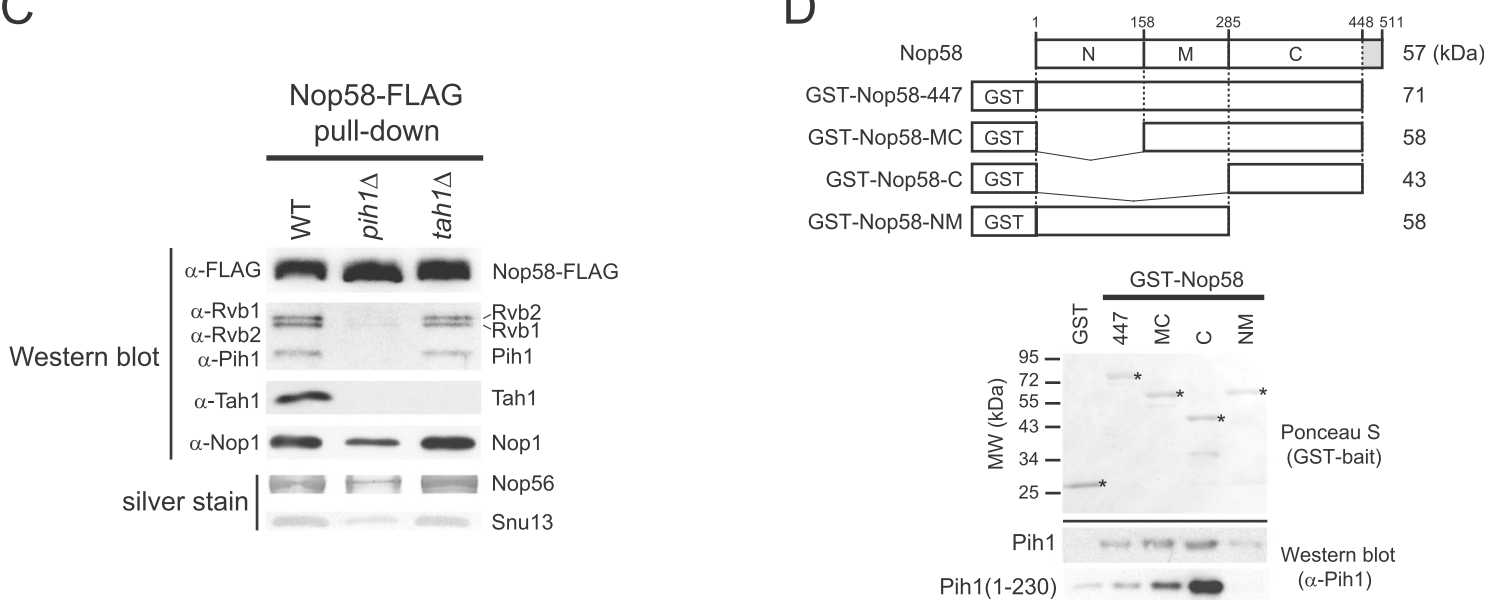

$E$

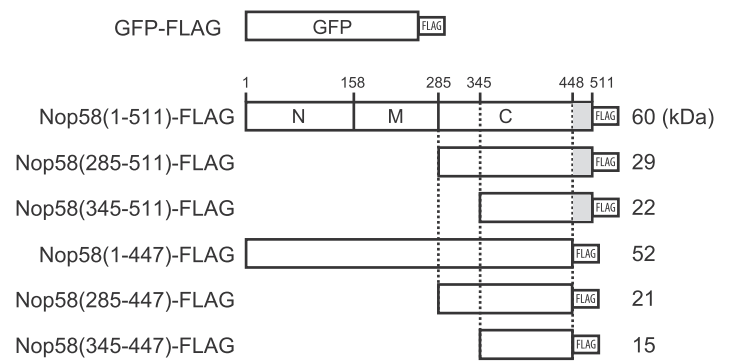

Figure 1 (See legend on next page.) 
(See figure on previous page.)

Figure 1 Interaction between the R2TP complex and Nop58. (A) Protein components of the R2TP and box C/D snoRNP complexes are shown on top. On bottom are the Western blot analyses for the presence of R2TP proteins in FLAG-tagged Nop1, Snu13, Nop56, and Nop58 pulldown complexes from log phase cells. Cell lysates from same wet weight of log phase cells were used for each pulldown. (B) Western blot and silver stain analysis of Nop58-FLAG pulldown complex purified from nuclease-treated or untreated cell lysates. Nop56 and Snu13 were identified by mass spectrometry. (C) Western blot analysis of R2TP complex interaction with Nop58-FLAG complex purified from wildtype, pih1 $\Delta$, or tah $1 \Delta$ background strains. (D) Schematic representations of Nop58 constructs generated for the in vitro binding assays are shown on top. The KKE/D charged region of Nop58 (residues 448-511) is shown in gray. In vitro binding assay for GST-Nop58 and Pih1 is shown on bottom. Ponceau $\mathrm{S}$ staining and Western blot analysis of GST pulldown assays using purified GST-Nop58 constructs incubated with Pih1 or Pih1(1-230). Stars indicate the location of the band of the GST-Nop58 construct being tested. (E) On the left are schematic representations of the Nop58-FLAG and GFP-FLAG constructs used for the pulldown assays from yeast cells. The KKE/D charged region (residues 448-511) of Nop58 is shown in gray. Western blot analysis of FLAG pulldown assays is shown on the right. Stars indicate the location of the band of Nop58-FLAG or GFP-FLAG constructs corresponding to the schematic on the left panel. The arrowhead shows the lane for Nop58(285-447)-FLAG construct which binds to R2TP but not snoRNP core proteins.

Deletion constructs lacking the N-terminal, N-terminal and middle, and C-terminal domains of GST-Nop58-447 were also generated and purified (Figure 1D, bottom panel). Since full-length purified Pih1 (344 amino acids) is unstable and readily aggregates, we also tested $\mathrm{N}$-terminal domain of Pih1, Pih1(1-230), which is more stable and soluble $[14,26,35]$. Both full-length Pih1, as well as, the Nterminal domain of Pih1(1-230) had the highest affinity for the C-terminal domain of Nop58, GST-Nop58-C, while weaker binding was detected with the other Nop58 constructs (Figure 1D).

To further analyze the interactions among R2TP, Nop58, and the other snoRNP factors in vivo, we generated FLAG-tagged Nop58 subdomain constructs in p416GAL vector for the overexpression of the constructs in yeast under GAL1 promoter. As shown in Figure $1 \mathrm{E}$ (left panel), we constructed six FLAG-tagged Nop58 constructs, as well as, GFP-FLAG construct as a negative control. All FLAG-fused proteins were overexpressed after galactose induction, except for Nop58(1-447)-FLAG, and each FLAG-tagged complex was co-immunopurified by using anti-FLAG beads (Figure 1E, right panel). The FLAG-tagged proteins that contain the KKE/D repeat, Nop58(1-511), Nop58(285-511), and Nop58(345-511), migrated slower than their actual molecular weight, which could be due to the relatively high content of positively charged lysine residues in their sequence. After the FLAG pulldown, the levels of interacting R2TP and box C/D snoRNP core proteins were compared by Western blot analysis. All R2TP and snoRNP proteins bound to fulllength Nop58(1-511), however, snoRNP proteins bound to Nop58 C-terminal domain (285-511) to a lesser extent, and both snoRNP and R2TP proteins did not interact significantly with Nop58(345-511). Intriguingly, R2TP remained associated with Nop58 C-terminal domain deleted of KKE/D (285-447) whereas the snoRNP proteins did not bind this construct. By comparing the construct of Nop58(285-511) with Nop58(285-447), the difference between the two is the presence or absence of KKE/D repeat, suggesting that the KKE/D region might contribute to the interaction between Nop58 and the other snoRNP core components (see Discussion). No binding was observed between Nop58(345-447) and R2TP or snoRNPs. This suggests that a main R2TP binding site in Nop58 is between residues 285-345 at the C-terminus of Nop58. Hence, in this analysis, we identified the Nop58(285-447) region as an exclusive binding site for the R2TP complex and not for snoRNP core proteins. This again strongly suggests that R2TP associates with unassembled Nop58 in vivo.

In summary, the data from Figures $1 \mathrm{~A}-\mathrm{E}$, indicate that the interaction between R2TP and Nop58 is primarily mediated by the binding of the $\mathrm{N}$-terminal domain of Pih1 to the C-terminal Nop domain of Nop58 and that the association does not require other snoRNP core proteins.

\section{The R2TP complex stabilizes Nop58}

To investigate the effect of the R2TP complex on the stability of the core box C/D snoRNP proteins, we assessed the steady-state levels of Nop58, Nop56 (FLAGtagged), Nop1, and Snu13 (FLAG-tagged) in WT, $r v b 1$-DAmP, $r v b 2-\mathrm{DAmP}$, pih1 $\Delta$, and tah1s log phase cells by Western blot analysis. The DAmP strains are hypomorphic alleles of $R V B 1$ and $R V B 2$ resulting in lower protein levels than in wildtype cells [36]. Intriguingly, only Nop58 protein levels were decreased in rvb1-DAmP, rvb2-DAmP, and more considerably in pih1 1 . Deletion of TAH1 had only a slight effect on Nop58 levels. Nop56, Nop1, and Snu13 protein levels were unaffected by the deletion or depletion of the other R2TP proteins (Figure 2A).

We further analyzed the Pih1-dependent stabilization of Nop58 using a conditional expression plasmid p416GAL-PIH1 in which Pih1 expression is under a galactose-inducible promoter. The plasmid was transformed into NOP56-FLAG pih1 1 cells and SNU13-FLAG pih $1 \Delta$ cells. Pre-cultures were grown in galactose minimal media plus raffinose for $16 \mathrm{~h}$ until stationary phase to overexpress Pih1, and then inoculated in glucose media to 


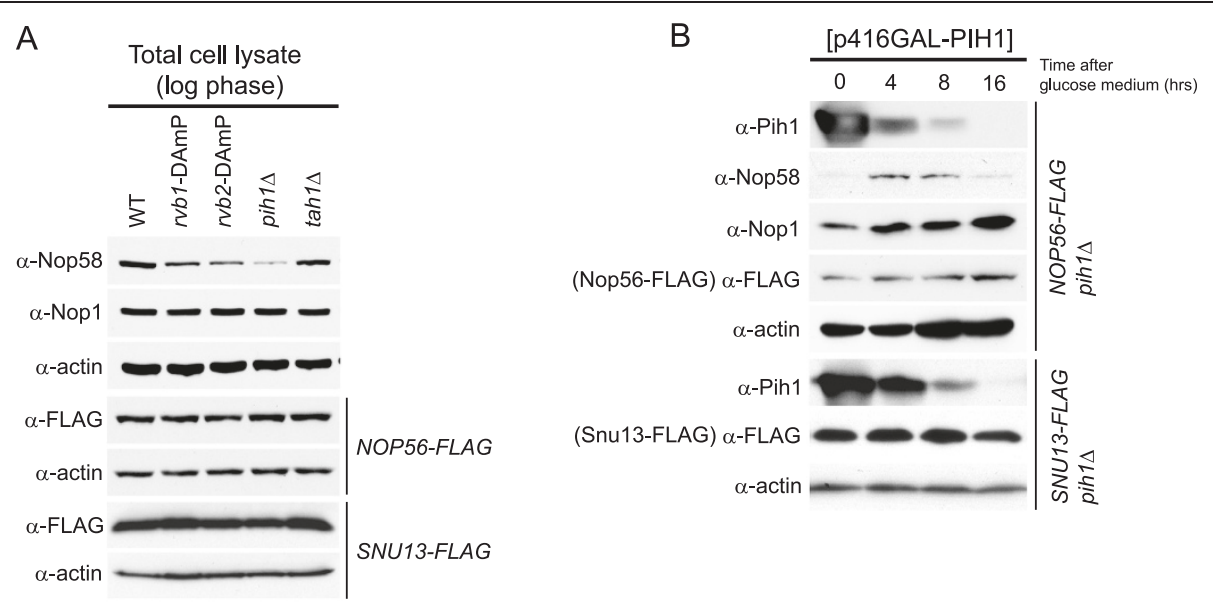

C

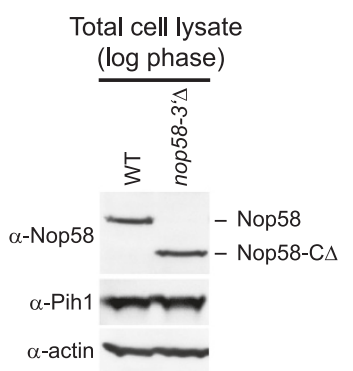

Figure 2 Effect of R2TP complex on Nop58 stability. (A) Western blot analysis of steady-state protein levels of box C/D snoRNP protein components in WT, rvb1-DAmP, rvb2-DAmP, pih1 1 , and tah1 $1 \Delta$ strains in log phase cells. (B) Western blot analysis of protein levels of box C/D snoRNP core proteins in NOP56-FLAG pih1 $\Delta$ and SNU13-FLAG pih1 1 strains transformed with p416GAL-PIH1 plasmid. Cells were shifted from galactose- to glucose-containing medium, and protein levels were analyzed as a function of time. (C) Western blot analysis of Nop58 and Pih1 steady-state levels in log phase WT and nop58-3's cells.

repress the expression of Pih1 from the plasmid. Since Nop1, Nop56, and Nop58 levels are significantly decreased in stationary phase (see below), we maintained the cells in log phase $\left(\mathrm{OD}_{600}\right.$ between 0.3 and 0.6$)$ by continuously adding fresh glucose medium to the culture approximately every $2 \mathrm{~h}$. After the glucose shift, Pih1 levels were drastically reduced over time. In contrast, Nop1, Nop56FLAG, and Nop58 levels initially increased until $4 \mathrm{~h}$ due to growth phase change from stationary to log phase (Figure 2B). After 4 h, Nop1, Snu13-FLAG, and Nop56FLAG levels remained constant over the time of the experiment, however, Nop58 levels gradually decreased as observed at 8 and $16 \mathrm{~h}$ after the shift (Figure 2B). This indicates that Pih1 is required for the stability of Nop 58 in vivo, but has no effect on the stability of Snu13, Nop1, or Nop56.

We also examined if Nop58 may regulate Pih1 stability by comparing Pih1 protein levels in WT and nop58-3' $\Delta$ strains. The latter strain, which shows a significant growth defect (data not shown and [14]), expresses a Nop58 mutant missing the last 83 amino acids (Nop58-C $\Delta$ ) that include part of the Nop domain. However, Pih1 levels were not affected by the functionally deficient Nop58 (Figure 2C).

\section{The R2TP complex does not interact with box C/D SnoRNA}

To investigate whether R2TP interacts with mature box C/D snoRNAs, we pulled down R2TP or box C/D snoRNP proteins (as a positive control) by performing Pih1-FLAG or Nop56-FLAG pulldowns, respectively. The presence of bound U14 snoRNA was detected by Northern blot analysis. Mature U14 snoRNA coimmunoprecipitated with Nop56-FLAG but not with Pih1-FLAG (Figure 3A).

To investigate the interaction between the R2TP complex and pre-snoRNA, we purified RNA from FLAG pulldown complexes of Rvb1, Rvb2, Pih1, and each of the box C/D snoRNP proteins (Nop1, Snu13, Nop56, and Nop58), and then performed RT-PCR using U14 pre-snoRNA specific primers. All box C/D snoRNP components associated with U14 pre-snoRNA, however, we were not able to detect the pre-snoRNA in Rvb1, Rvb2, and Pih1-FLAG pulldown complexes (Figure 3B). 
A

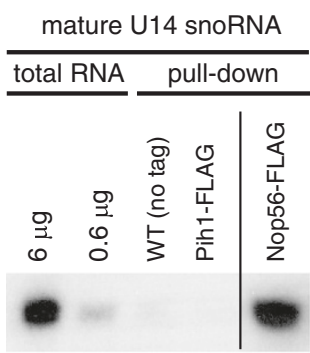

B

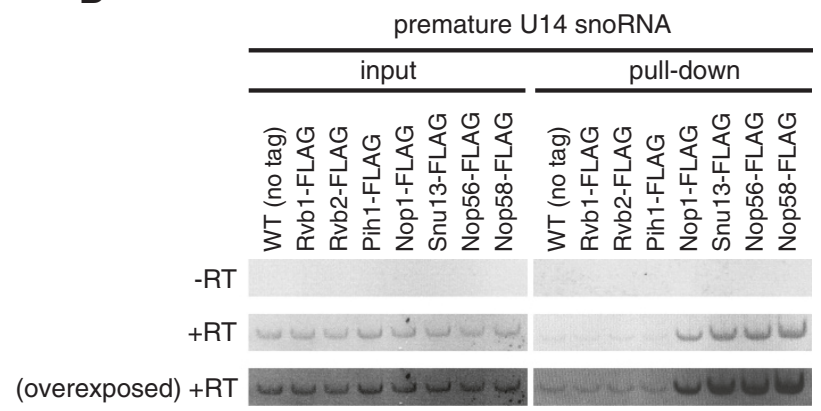

Figure 3 Analysis of R2TP complex interaction with mature and premature box C/D snoRNA. (A) Northern blot analysis of mature U14 snoRNA in pulldown complexes of Pih1-FLAG (R2TP) and Nop56-FLAG (box C/D snoRNP); $6 \mu \mathrm{g}$ and $0.6 \mu \mathrm{g}$ of total RNA and RNAs purified from the pulldown complexes are shown. WT (no tag) strain was used as a negative control. (B) RT-PCR analysis of premature U14 snoRNA in pulldown complexes of R2TP (Rvb1-, Rvb2-, and Pih1-FLAG) and box C/D snoRNP (Nop1-, Snu13-, Nop56-, and Nop58-FLAG). RNAs were purified from the pulldown complexes and PCR was performed with (+RT) or without (-RT) reverse transcription.

These experiments suggest that the R2TP complex might not significantly interact with mature or premature snoRNA and might not be directly involved in snoRNA processing or maturation.

\section{The interaction of R2TP with Nop58 is modulated by nucleotide binding}

Given that Rvb1 and Rvb2 are AAA + superfamily proteins and have ATPase activity [37] that is known to be essential for snoRNA biogenesis [38], it is very likely that R2TP complex function in box C/D snoRNP assembly is regulated by ATP binding and/or hydrolysis. To investigate the effect of nucleotide on the binding of R2TP to box C/D snoRNP complex, we pulled down the Nop58FLAG complex from log phase yeast cells using antiFLAG beads, which also brings down R2TP as shown in Figure 1. The bead-bound complex was then incubated for $30 \mathrm{~min}$ at $30^{\circ} \mathrm{C}$ in the presence or absence of $4 \mathrm{mM}$ ATP. In the presence of ATP, most of snoRNP-bound Rvb1/2 proteins were released into the supernatant; Pih1 and Tah1 were also released but to a lesser extent, while Nop58 and the other box C/D proteins remained largely bound to the beads (Figure 4A). Similar results were obtained using ADP and ATPYS (not shown).

To determine whether the addition of nucleotide to R2TP causes the disassembly of the complex, we immunopurified R2TP from Pih1-FLAG strain using antiFLAG beads, and then the bead-bound complex was incubated in the absence or presence of $4 \mathrm{mM}$ ADP, ATP, or ATP $\gamma$ S. The levels of the protein components on the beads or in the supernatant fraction were determined by Western blot (Figure 4B). The bait Pih1FLAG remained tightly retained on the anti-FLAG beads, whereas basal amounts of Tah1 were present in all supernatant fractions, suggesting that the release of Tah1 is not nucleotide dependent and could be a dilution effect. However, Rvb1/2 were dissociated from Pih1-FLAG in the presence of ADP, ATP and ATP $\gamma$ S, indicating that the dissociation of Rvb1/2 from Pih1/Tah1 was induced by nucleotide binding rather than ATP hydrolysis.

Next, we attempted to reconstitute complexes in vitro, Nop58 C-terminal domain (residues 285-447) with Rvb1/2 and Nop58 C-terminal domain with R2TP, using purified recombinant proteins from E. coli. As shown in Figure 4C, both Rvb1/2 and R2TP formed complexes with glutathione bead-bound GST-Nop58-C, indicating that Rvb1/2 alone is also able to bind the C-terminal domain of Nop58. By using the GST-Nop58-C/R2TP complex, we tested whether nucleotides release the R2TP complex from Nop58 C-terminal domain as observed in Figure 4A. Indeed, R2TP was released from an in vitro reconstituted GST-Nop58-C/R2TP complex upon addition of ADP, ATP, and ATP $\gamma$ S (Figure 4D).

Taken together, the results of Figure 4 demonstrate that nucleotide binding to R2TP dissociates the R2TP complex itself and also releases R2TP from Nop58 Cterminal domain.

\section{The interaction between the R2TP complex and Nop58 is dependent on cell growth phase}

It has been shown that growth phase and nutrient availability actively regulate ribosome biosynthesis including the expression of pre-rRNAs, r-protein genes, and ribosomal biogenesis (Ribi) genes [39]. Given that snoRNP biogenesis is tightly linked with ribosome synthesis, it is reasonable to expect that snoRNP biogenesis is also coordinated with the growth phase of the cell. Therefore, we initially determined the steady-state protein levels of core box C/D snoRNP and R2TP components in log and stationary phase cells. As shown in Figure 5A, Snu13FLAG, Rvb1, Rvb2, and Pih1 levels did not significantly change between log and stationary phase; however, Nop1, Nop56-FLAG, and Nop58 levels significantly decreased in stationary phase compared to log phase. On the other 
A

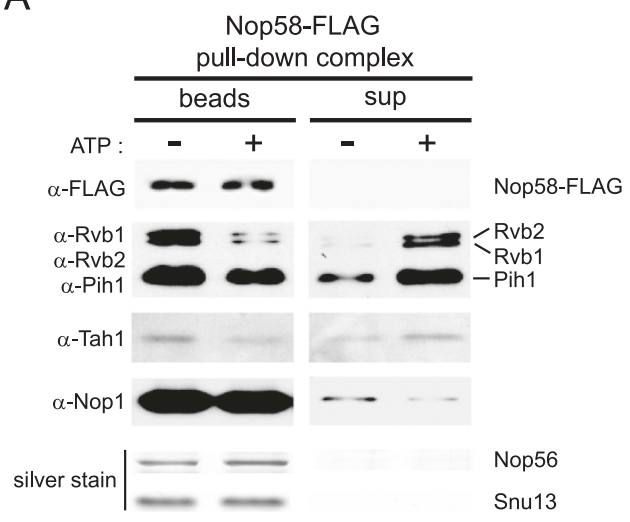

B

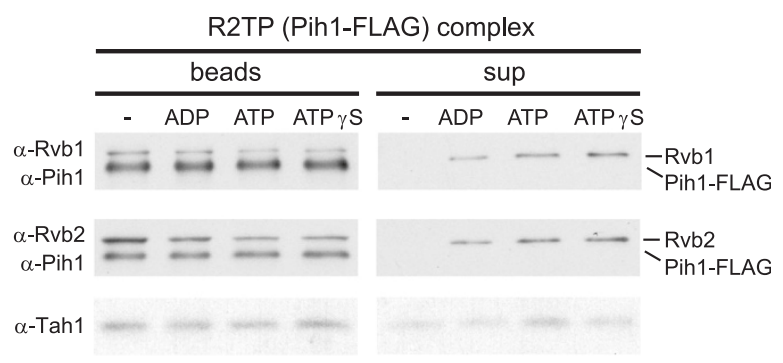

C

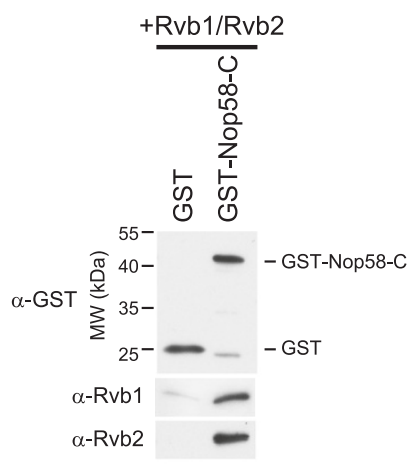

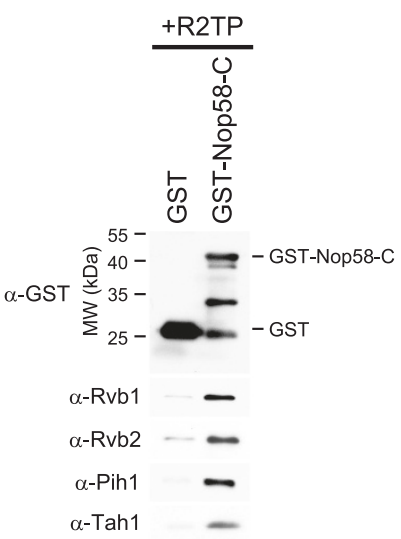

D

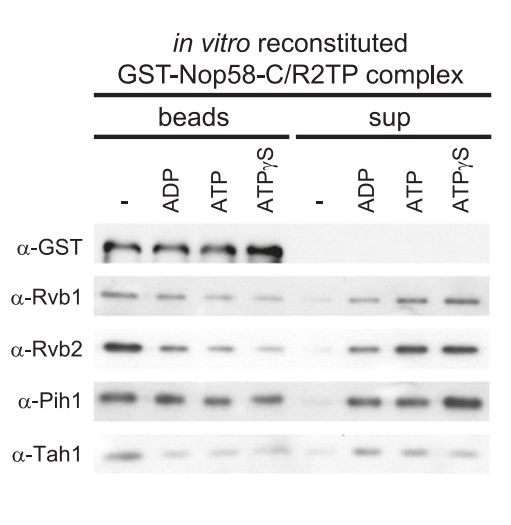

Figure 4 Effect of nucleotides on the interaction between Nop58 and R2TP. (A) Western blot or silver stain analysis of Nop58-FLAG pulldown complex in the absence or presence of ATP. The Nop58-FLAG complex purified on a-FLAG beads from cell lysates was incubated with $4 \mathrm{mM}$ ATP for $30 \mathrm{~min}$ at $30^{\circ} \mathrm{C}$. Supernatant and bead-bound fractions were, subsequently, analyzed. Nop58-FLAG, Rvb1, Rvb2, Pih1, Tah1, and Nop1 were detected by Western blot, while Nop56 and Snu13 were detected by silver stain (and identified by mass spectrometry). (B) Western blot analysis of the R2TP (Pih1-FLAG) complex in the absence or presence of different nucleotides. The R2TP complex purified on a-FLAG beads from cells having Pih1 endogenously FLAG-tagged was incubated in the absence or presence of $4 \mathrm{mM} \mathrm{ADP}$, ATP, or ATPYS for $30 \mathrm{~min}$ at $30^{\circ} \mathrm{C}$. Supernatant and bead-bound fractions were, subsequently, analyzed. (C) In vitro binding assay for Nop58 C-terminal domain with Rvb1/2 or R2TP complex. GST alone or GST-Nop58-C (residues 285-447) were bound to glutathione-beads and incubated with Rvb1/2 or R2TP complex. After washing the beads, proteins retained on the beads were detected by Western blot analysis. (D) Western blot analysis of in vitro reconstituted GST-Nop58-C/R2TP complex in the absence or presence of different nucleotides. The GST-Nop58-C/R2TP complex bound to beads prepared as in Figure $4 \mathrm{C}$ (right panel) was incubated in the absence or presence of $5 \mathrm{mM} \mathrm{ADP}$, ATP, or ATPYS for $30 \mathrm{~min}$ at $30^{\circ} \mathrm{C}$. Supernatant and bead-bound fractions were, subsequently, analyzed.

hand, Tah1 levels were increased in stationary phase relative to $\log$ phase (Figure $5 \mathrm{~A}$ ) and this might be related to the role of Tah1 in stress response as an Hsp90 co-factor in addition to its role with the R2TP complex [21].

To test whether the interaction between the R2TP complex and Nop58 is affected by the cell's growth phase, we performed pulldown assays of Nop58-FLAG from log and stationary phase cells. In this experiment, because of the different levels of the snoRNP proteins between log and stationary phases (Figure 5A), we prepared Nop58FLAG cell lysates from equal weight $(1 \mathrm{~g})$ of $\log$ and stationary phase cell pellets but also from three times more by weight $(3 \mathrm{~g})$ of stationary phase cells to obtain comparable levels of Nop58-FLAG pulldown complexes. Cell lysates from untagged wildtype cells were also prepared the same way and used as a negative control. As shown in Figure 5B inset, comparable levels of Snu13, Nop1, and Nop56 associated with Nop58 in both log and stationary phases. The interaction of R2TP with Nop58FLAG was evident in log phase but was significantly reduced in stationary phase and stationary phase $(\mathrm{x} 3)$ (Figure 5B), suggesting that the interaction between 


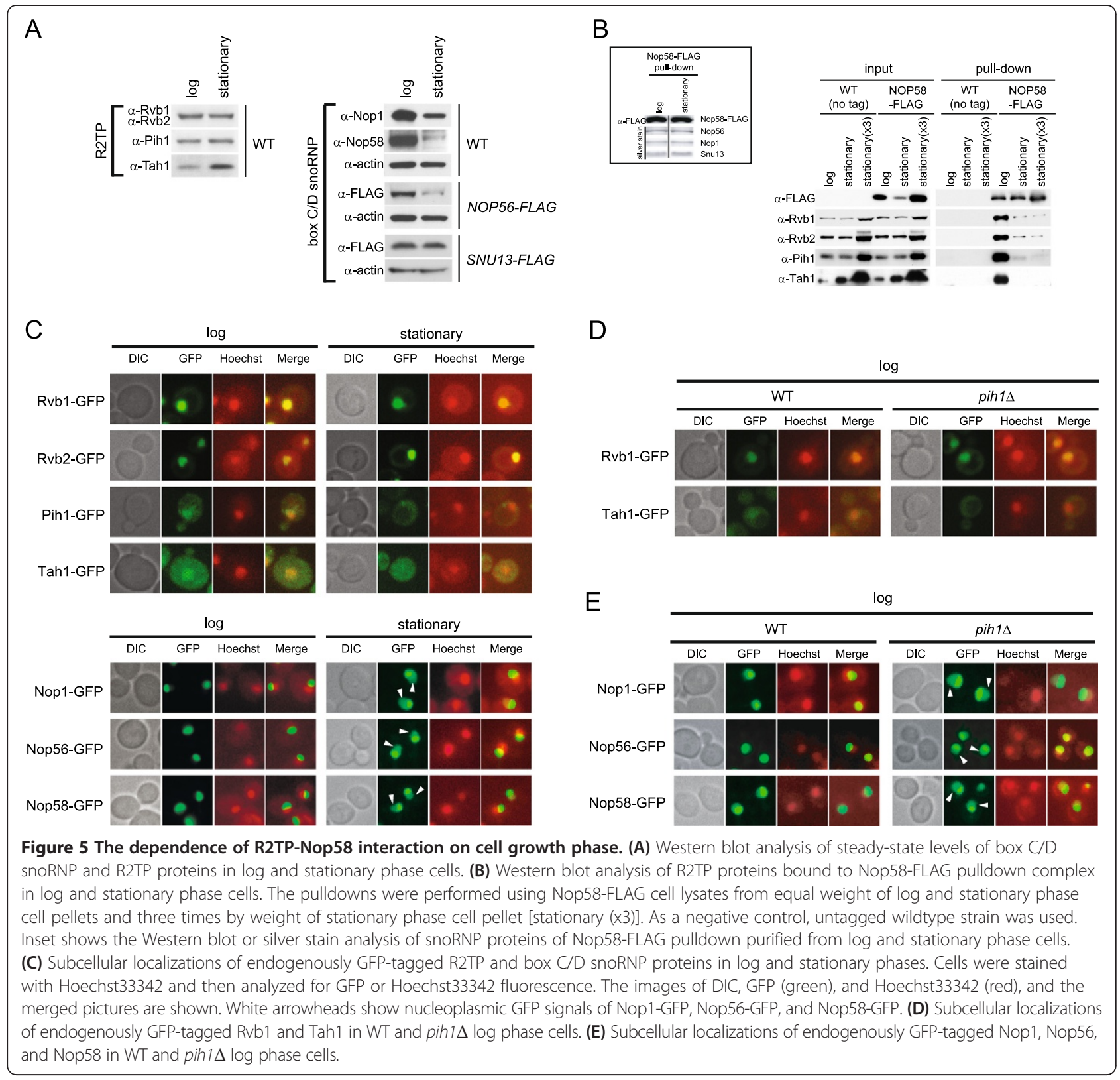

the R2TP complex and Nop58 is controlled by cell growth phase or that there is less free pool of Nop58 in stationary phase cells.

To determine how the interaction between R2TP and box C/D snoRNP is reduced in stationary phase, we next examined the subcellular localization of the protein components of the R2TP and box C/D snoRNP complexes using endogenously C-terminally GFP-tagged strains. Interestingly, all subunits analyzed in this experiment showed significant subcellular localization changes between $\log$ and stationary phases (Figure $5 \mathrm{C}$ and Additional file 1: Figure S1). Rvb1-GFP and Rvb2-GFP mainly localized in the nucleus and to a lesser extent to the cytoplasm during log phase as well as stationary phase. Furthermore, we noticed that Rvb1-GFP and Rvb2-GFP form cytoplasmic foci in stationary phase of some cells (Additional file 1: Figure S1). Intriguingly, both Pih1-GFP and Tah1GFP localized in the nucleus and cytoplasm in log phase, and distributed to the cytoplasm in stationary phase (Figure 5C and Additional file 1: Figure S1). Nop1GFP, Nop56-GFP, and Nop58-GFP showed dense localization in the nucleolus in log phase, however, the GFP signals diffused into the nucleoplasm in stationary phase. Quantification of the fluorescence signals, showed that $10 \%$ to $17 \%$ of the box C/D snoRNP proteins can be detected in the nucleoplasm in log phase and that the number increases to $30 \%$ to $35 \%$ in stationary phase (refer to Figure 6D). This indicates that there is a defect or 


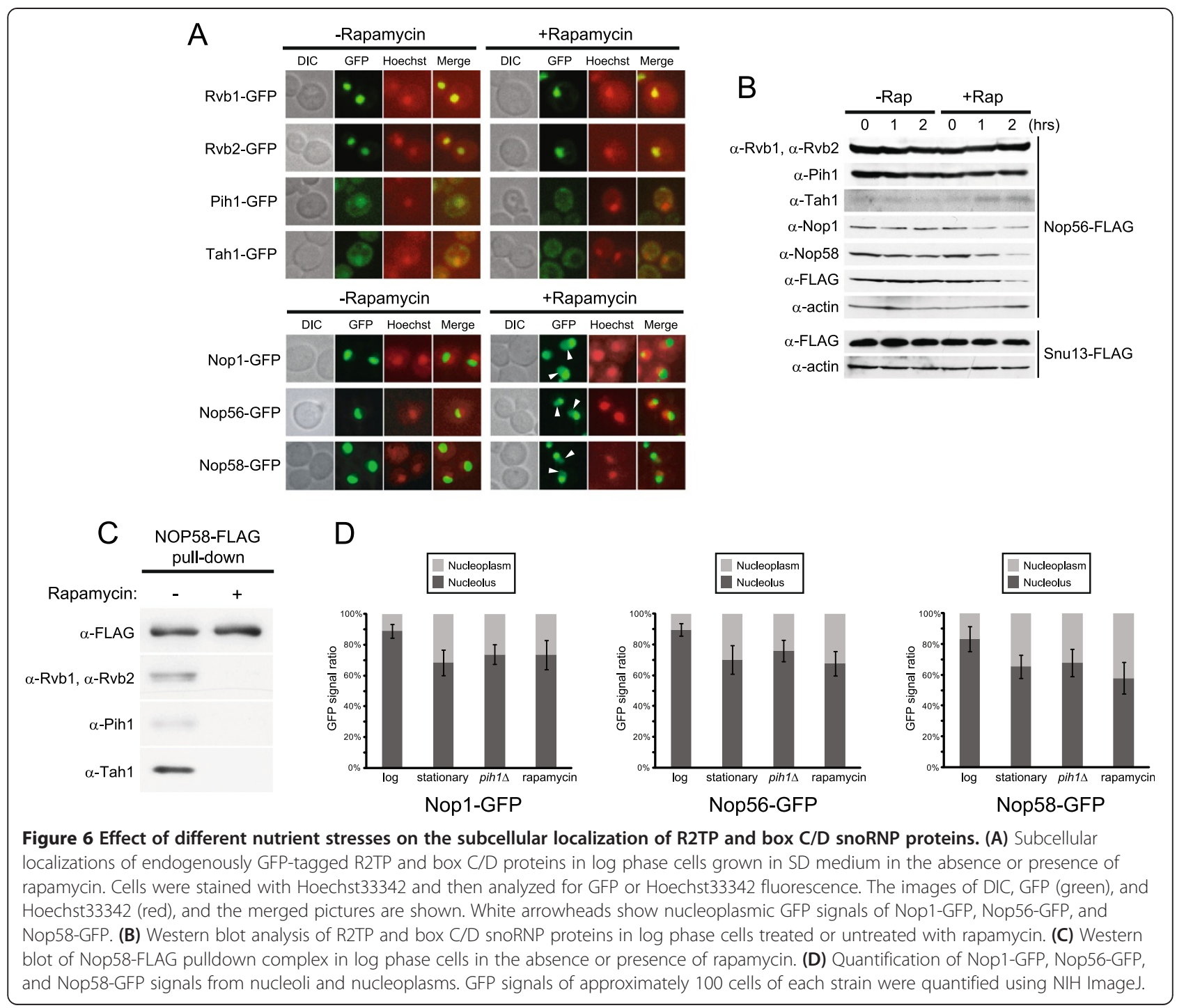

reduction in the rate of translocation of the snoRNP proteins from the nucleoplasm to the nucleolus in stationary phase. Note that we could not detect a reliable signal for Snu13-GFP. Hence, the subcellular localizations of the R2TP and snoRNP components dynamically changes based on growth phase, which could explain the growth phase-dependent interaction between R2TP and Nop58 (Figure 5B).

\section{Pih1 is required for the proper localization of R2TP and box C/D snoRNP proteins}

In previous studies, it was shown that Rvb2 depletion as well as PIH1 deletion caused delocalization of snoRNP proteins [25,38]. Since Pih1 seems to act as an adaptor between R2TP and Nop58 (Figure 1C,E), we examined how Pih1 might affect R2TP and snoRNP localizations by observing GFP fusion proteins of all related components in pih1 $1 \Delta$ background. In log phase pih1 $\Delta$ cells, less Tah1-GFP was present in the nucleus compared to WT cells (Figure 5D and Additional file 2: Figure S2). On the other hand, there was no significant change in Rvb1-GFP localization between WT and pih1s cells (Figure 5D and Additional file 2: Figure S2). This could reflect the fact that Rvb1/2 are involved in many other nuclear complexes in addition to R2TP [24] and that the levels of Rvb1/2 in the cell are much higher than that of Pih1 (unpublished data). Interestingly, the deletion of PIH1 led to increased nucleoplasmic localization of Nop1, Nop56, and Nop58 (Figure 5E, Figure 6D, and Additional file 3: Figure S3), which is consistent with the proposed role of Pih1 in the nucleolar localization of box C/D core proteins [25]. These results suggest that Pih1 maintains the nuclear localization of R2TP and is required for the proper localization of the box C/D snoRNPs in the nucleolus. 


\section{Nutrient starvation affects the subcellular localization of R2TP and box C/D snoRNP proteins}

The above localization results led us to investigate the subcellular distribution of the R2TP and box C/D snoRNP proteins in response to different nutrient starvation conditions such as carbon starvation, nitrogen starvation, and rapamycin treatment. Rapamycin inactivates the TOR nutrient signaling pathway and induces a starvation-like state in yeast [40]. As in stationary phase cells (Figure 5C), Pih1 and Tah1 delocalized to the cytoplasm from the nucleus upon rapamycin treatment, while Nop1, Nop56, and Nop58 diffused into the nucleoplasm from the nucleolus (Figure 6A,D). Similar relocalization patterns were observed under carbon and nitrogen starvation conditions (Additional file 4: Figure S4). Rvb1 and Rvb2 showed no drastic changes in their localizations under these conditions consistent with the results of Figure $5 \mathrm{C}$.

Levels of all box C/D snoRNP proteins, except for Snu13, decreased upon rapamycin treatment (Figure 6B), as seen in stationary phase cells (Figure $5 \mathrm{~A}$ ). Also, the interaction between R2TP and Nop58-FLAG significantly decreased in rapamycin-treated cells (Figure 6C). The results of Figures 5 and 6 suggest that nutrient starvation destabilizes the box C/D snoRNP complex as a result of the dynamic nucleo-cytoplasmic translocation of the R2TP complex, which could consequently reduce the levels of box C/D snoRNPs translocated or the rate of translocation from the nucleoplasm to the nucleolus.

\section{Subcellular localization of the R2TP complex is dependent on nucleo-cytoplasmic transport system}

We asked whether the dynamic translocation of R2TP complex in different growth phases and nutrient conditions is dependent on the nucleo-cytoplasmic transport system. First, we examined the dependence of Pih1 and Tah1 nuclear import on Kap121, which is one of the major karyopherins involved in nuclear import [41]. The localization of endogenously C-terminally GFP-tagged Pih1 and Tah1 were analyzed in wildtype and kap121-34 temperature sensitive mutant allele at the permissive $\left(26^{\circ} \mathrm{C}\right)$ and restrictive temperatures $\left(30^{\circ} \mathrm{C}\right)$. Cells were grown to early-log phase at the permissive temperature and further incubated for $2 \mathrm{~h}$ at $26^{\circ} \mathrm{C}$ or $30^{\circ} \mathrm{C}$. In wildtype cells, Pih1-GFP and Tah1-GFP localized mainly in the nucleus with some distribution of GFP-tagged proteins in the cytoplasm at both $26^{\circ} \mathrm{C}$ and $30^{\circ} \mathrm{C}$ (Figure 7A). In kap121-34 mutant, similar localizations of Pih1-GFP and Tah1-GFP were observed at $26^{\circ} \mathrm{C}$ as in wildtype cells, however, both Pih1-GFP and Tah1-GFP delocalized to the cytoplasm upon inactivation of Kap121 at $30^{\circ} \mathrm{C}$ (Figure 7A). Hence, Kap121 mediates the nuclear import of Tah1 and Pih1. To further confirm this result, a parallel experiment was carried out using cells overexpressing either Nup53, which is known to significantly inhibit the
Kap121-mediated nuclear import pathway, or Nup53$\mathrm{C} \Delta$, which cannot inhibit Kap121 activity since it lacks the Kap121 binding domain (residues 405-430) [42]. Consistent with the above results, overexpression of Nup53 greatly inhibited the nuclear import of both Pih1-GFP and Tah1-GFP, whereas the overexpression of Nup53-C $\Delta$ did not inhibit the translocation (Figure 7B), showing that the nuclear import of Pih1 and Tah1 is Kap121-dependent. We did not observe a significant change in the subcellular localization of Rvb1-GFP and Rvb2-GFP in Nup53-C $\Delta$ vs. Nup53 overexpressing cells (Additional file 5: Figure S5). This might reflect the fact that a large amount of Rvb1 and Rvb2 are part of other complexes such as Ino80 and SWR-C chromatin remodeling complexes in the nucleus and, hence, their nuclear import might be independent of Kap121 activity. However, pulldown of the R2TP complex using Pih1-FLAG from Nup53-C $\Delta$ and Nup53 overexpressing cells at log phase showed no changes in the stoichiometry of the complex (Figure 7C) suggesting that all the R2TP protein components tightly form a complex and translocate together between the nucleus and cytoplasm.

Next, we analyzed the dependence of R2TP complex export on Crm1, which is one of the main exportins of ribosomes in eukaryotes [43,44]. To inhibit the activity of Crm1, we used cells expressing crm1-T539C mutant, which is sensitive to leptomycin B (LMB), an inhibitor of Crm1 [45]. In these cells, Pih1-GFP and Tah1-GFP delocalized to the cytoplasm from the nucleus in stationary phase cells in the absence of LMB (Figure 7D) as seen in Figure $5 \mathrm{C}$, however, the two proteins remained in the nucleus in the presence of LMB (Figure 7D). Thus, our observations suggest that the dynamic nucleo-cytoplasmic translocation of the R2TP complex in different growth phases is dependent on the karyopherins, Kap121, and Crm1. Note that we were not able to detect direct interactions between Kap121 or Crm1 and R2TP (data not shown), which suggests that R2TP localization is indirectly regulated by the respective importin and exportin or that the interactions are weak.

\section{Nuclear localization of R2TP is important for proper cell growth}

To investigate whether the nuclear-cytoplasmic shuttling of R2TP has an effect on cell growth, we prevented or reduced R2TP shuttling by adding a nuclear export signal (NES) or a nuclear localization signal (NLS) to the Pih1 sequence. We generated constructs which express Pih1-GFP fused to the NES derived from human protein kinase A inhibitor (LALKLAGLDINKT) or to the NLS from SV40 T antigen (PKKKRKV) [46] controlled by PIH1 native promoter. As controls, plasmids expressing Pih1-GFP with non-functional mutant NES (LALKLAGADTNKT) or NLS (PKAKRKV) denoted as mNES and mNLS, respectively, were also used [46]. The constructs 


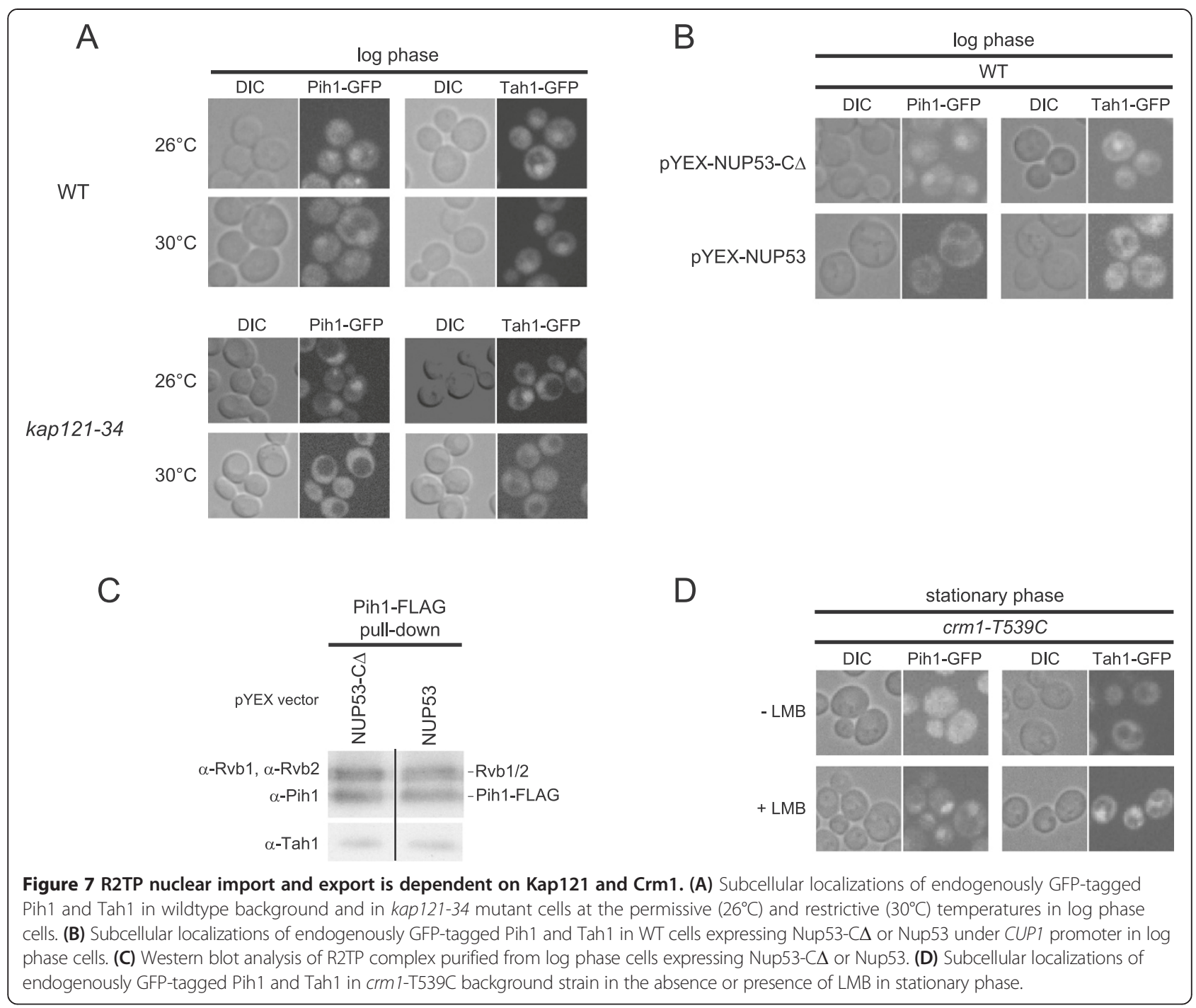

were transformed into Nop58-FLAG pih1 $1 \Delta$ background strain and GFP localization was observed using fluorescence microscopy. Pih1-GFP-NES and Pih1-GFP-NLS remained in the cytoplasm and in the nucleus, respectively, in both $\log$ and stationary phases (Figure 8A). On the other hand, Pih1-GFP-mNES and Pih1-GFP-mNLS changed localization between the nucleus in log phase and the cytoplasm in stationary phase (Figure $8 \mathrm{~A}$ ) as observed for untagged Pih1-GFP (Figure 5C).

To analyze the effect of forcing Pih1 localization to the cytoplasm or nucleus, we compared cell growth of different transformants. Intriguingly, whether on plates or in liquid culture, we observed a slower growth phenotype for pih $1 \Delta$ cells expressing Pih1-GFP-NES compared to pih1 1 cells expressing Pih1-GFP-NLS, Pih1-GFPmNES, or Pih1-GFP-mNLS (Figure 8B,C). We did not observe a faster growth or delay in entering stationary phase for the Pih1-GFP-NLS transformant compared to Pih1-GFP-mNLS (Figure 8C inset). Also, Nop58-FLAG protein levels were decreased in both log and stationary phase in pih1 $\Delta$ cells expressing Pih1-GFP-NES (or in cells having vector only) compared to other transformants. It should also be noted that Pih1-GFP-NLS does not restore the levels of Nop58-FLAG present in stationary phase to those observed in log phase (Figure 8D). This suggests that other additional cellular mechanisms control Nop58 levels in stationary phase.

In summary, the results of Figure 8 suggest that the nuclear localization of the R2TP complex is required for its proper function in maintaining Nop58 stability and in controlling box C/D snoRNP biogenesis.

\section{Discussion}

Our data clearly demonstrate that the R2TP complex plays an essential role in stabilizing Nop58 in the process of box C/D snoRNP maturation (Figures 1 and 2). Intriguingly, this R2TP function is influenced by growth phases and nutrient conditions and is regulated by 


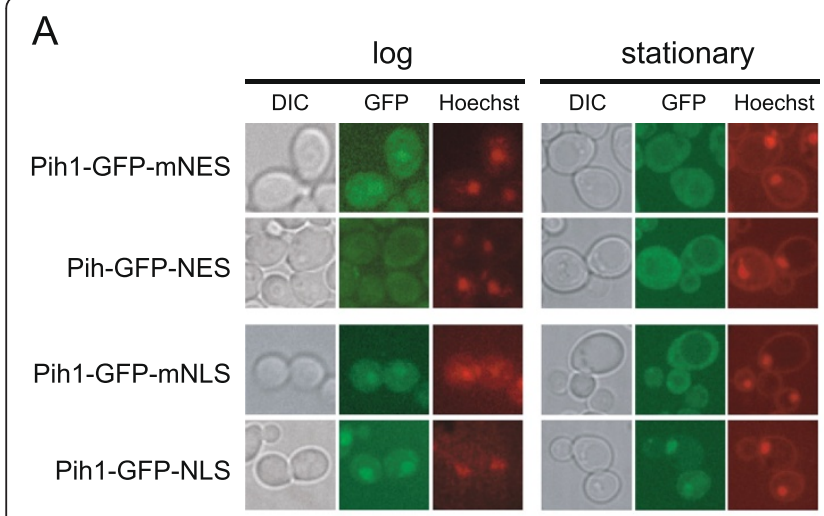

C

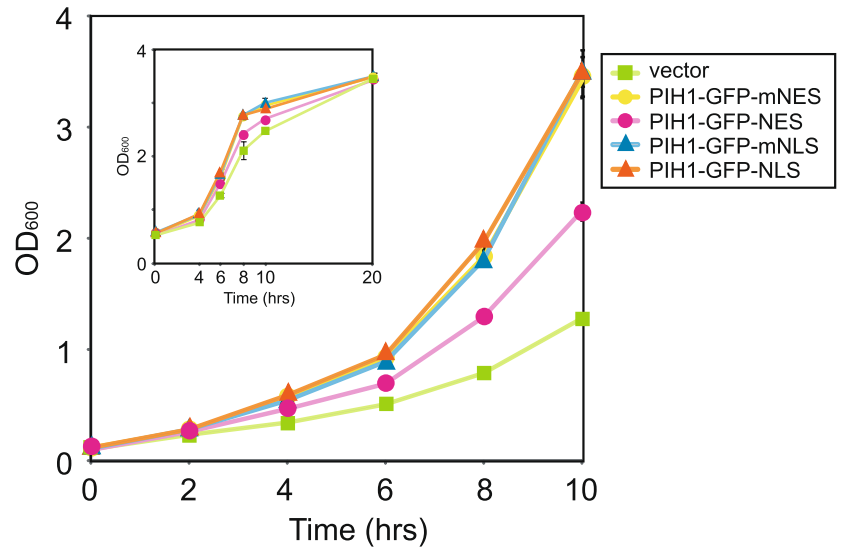

B

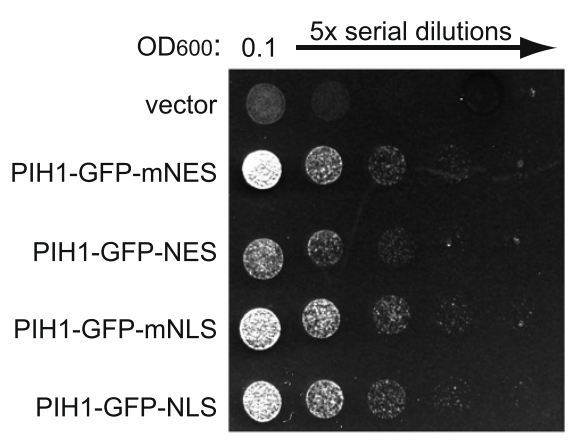

$37^{\circ} \mathrm{C}, 2$ days

D

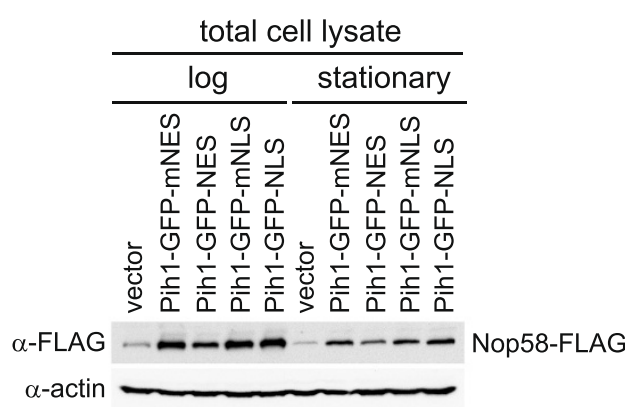

Figure 8 Effect of targeting Pih1 to cytoplasm or nucleus on cell growth. (A) Pih1-GFP was fused with nuclear export signal (NES), mutagenized nuclear export signal (mNES), nuclear localization signal (NLS), or mutagenized nuclear localization signal (mNLS), and expressed from pRS315 vector under the PIH1 promoter in Nop58-FLAG pih1 1 strain. Cells were stained with Hoechst33342 and then analyzed for GFP or Hoechst33342 fluorescence. The images of DIC, GFP (green), and Hoechst33342 (red) are shown. (B) Dilution spot assay of the strains described in A. Cells were serially diluted fivefold, spotted onto SD-Leu plate, and incubated for 2 days at $37^{\circ} \mathrm{C}$. (C) Growth curves in liquid culture for the different indicated strains. Cells were grown in SD-Leu medium at $37^{\circ} \mathrm{C}$ and $\mathrm{OD}_{600}$ was measured every $2 \mathrm{~h}$. Inset shows growth curve of the same strains until stationary phase. (D) Western blot analysis of total cell lysate of the different transformants. Cell lysates were prepared from cells grown at $37^{\circ} \mathrm{C}$ until log or stationary phase.

the subcellular localization of its protein components (Figures 5, 6, and 7).

The depletion or deletion of the R2TP components, except for Tah1, results in reduced Nop58 levels (Figure 2A and $\mathrm{B})$. This result suggests that the main functional components of the R2TP complex for snoRNP biogenesis are Rvb1, Rvb2, and Pih1, while Tah1 could be an accessory protein. This is consistent with our previous result showing that Tah1 effect on snoRNP biogenesis is subtle, and that Tah1 functions with Hsp90 to maintain Pih1 protein stability [14]. Although we observed a direct interaction of Rvb1/2 with Nop58 C-terminal domain in vitro (Figure 4C, left panel), the Nop58-FLAG pulldown experiment in pih1 $\Delta$ showed a significant reduction of Rvb1/2 binding with Nop58, indicating that the interaction of the R2TP complex with Nop58 is largely mediated by Pih1 in vivo (Figure 1C). More specifically, we found that the Nterminal domain of Pih1, residues 1-230, binds to the C- terminal Nop domain of Nop58 (Figure 1D). Furthermore, Pih1(1-230) was also found to interact with Rvb1/2, but the interaction was significantly enhanced for Pih1(1-248) [35]. We consider Pih1 to define the R2TP complex in yeast.

We found that the R2TP complex significantly interacts with the C-terminal domain of Nop58 spanning residues 285-447 (Figure 1E). No binding of snoRNP core proteins to this region of Nop58 was detected suggesting that R2TP interacts with Nop58 that has not been assembled with other snoRNP core factors. Unexpectedly, it was also observed that deletion of the KKE/D region from Nop58 C-terminus (residues 448-511) significantly reduced Nop58 interaction with the other snoRNP core proteins, suggesting that this charged region of Nop58 might contribute to the association between Nop58 and other snoRNP core factors. In yeast, the deletion of KKE/D does not significantly affect cell growth and box 
C/D snoRNA binding but causes a loss of the compaction of the nucleolus [47-49]. Also, it has been found that this highly charged domain of Nop58 interacts with Tgs1 in vitro; Tgs1 is a tri-methylguanosine synthase and is essential for hypermethylation of the $5^{1} \mathrm{~m}^{7} \mathrm{G}$ cap of snRNAs and snoRNAs [50]. Furthermore, in mammalian cells, the C-terminal domain of Nop58 containing the charged region is essential for the nucleolar localization of Nop58 and functions as a nucleolar localization signal (NoLS) [20]. Taken together, these observations suggest an important role for the KKE/D region of Nop58 in snoRNP biogenesis. It would be important to further analyze the physiological role of this domain.

If the yeast and archaeal box C/D complexes have the same general arrangement [33,34], then the Nop domain of Nop58 has multiple binding partners including the snoRNA, Snu13, and Pih1. Furthermore, based on the recent X-ray crystal structure of the archaeal box C/D RNA-protein complex [34], it is clear that Nop58 is a key component of the complex providing an interaction platform for the other core proteins and for the snoRNA.

Recently, it has been reported that Pih1-Tah1 heterodimer interacts with Snu13-U14 snoRNA through Rsa1 in vitro [51]. However, we were not able to identify an interaction between Snu13 and Pih1-Tah1 in vivo (Figure 1A). We also performed Rsa1-FLAG pulldowns using yeast soluble cell lysate but no interaction with R2TP components was detected (data not shown), suggesting that the Pih1-Tah1-Snu13-snoRNA-Rsa1 interaction might be weak or transient under physiological condition.

Intriguingly, the R2TP complex interacts with the unassembled form of Nop58 with high affinity (Figure 1A, $B, E)$, but does not interact with either mature or premature box C/D snoRNA (Figure 3). These results suggest that R2TP is involved in a very early stage of the box C/D snoRNP biogenesis before Nop58 assembles with the other snoRNP components: Snu13, Nop1, Nop56, and snoRNAs. In a previous study, we showed that the deletion or depletion of R2TP components affects the accumulation of mature box C/D snoRNAs [14]. This phenotype could be the result of the destabilization of Nop58. The interaction between R2TP and Nop58 is evident in log phase cells that require high production rate of snoRNPs for efficient ribosome biogenesis but not in stationary phase cells. Therefore, it is likely that the total box C/D snoRNP levels in the cell, and consequently pre-rRNA processing, could be effectively regulated by modulating Nop58 levels rather than other box C/D snoRNP core components. This would explain why the R2TP complex, by affecting the stability of Nop58, plays a critical role in cell physiology. Previously, we reported that R2TP is involved in snoRNP biogenesis in both log and stationary phases [14]. As mentioned above, we observed a strong interaction between R2TP and Nop58 in log phase whereas it was weaker in stationary phase. However, this low level of binding could be sufficient in stationary phase cells to maintain the basal levels of Nop58 and, subsequently, conserve snoRNA production.

In mammalian cells, it has been reported that the treatment of human cells by proteasome inhibitor MG132 leads to improper localization of Nop58 [52]. Furthermore, post-translational modifications of mammalian Nop58 have also been reported [53,54]. The protein has been found to be phosphorylated by casein kinase II (CK2), and, subsequently, SUMOylated to increase its stability. These observations further highlight the importance of regulating Nop58 stability. It will be interesting to identify such modifications for yeast Nop58 and to determine how the modifications affect Nop58 stability, function, and Nop58-R2TP interaction in response to different growth and nutrient conditions.

Based on the localization studies (Figures 5C, 6A, and $7 \mathrm{~A}, \mathrm{~B}, \mathrm{D})$, we unexpectedly found that the ability of the R2TP complex to modulate the assembly of box $\mathrm{C} / \mathrm{D}$ snoRNPs is regulated by a nucleo-cytoplasmic shuttling mechanism that is dependent on the Kap121 and Crm1 karyopherins, which are also known to be involved in nucleo-cytoplasmic trafficking of ribosomal proteins [41,43]. The shuttling of the R2TP proteins between the nucleus and the cytoplasm is growth phase and nutrient dependent. In stationary phase cells (Figure 5C) or in the absence of carbon or nitrogen sources (Additional file 4: Figure S4), or in the presence of rapamycin (Figure 6A), we observed that some of the R2TP complex shuttles out of the nucleus, all core box C/D snoRNP protein levels, except for Snu13, are reduced (Figure 5A and 6B) and a larger proportion of the snoRNP proteins are present in the nucleoplasm (Figures 5C, 6A, and Additional file 4: Figure S4). In contrast, when nutrients are available, then the R2TP complex is enriched in the nucleus (Figure 5C), all core box C/D snoRNP protein levels were recovered (Figures 5A and 6B) and localized in the nucleolus (Figure 5C). As shown in Figure 8, this shuttling of the R2TP complex actively influences cell growth. It is known that ribosome synthesis is the major energy consuming process in the cell [2], and that ribosome activity is tightly coupled to growth phase and nutrient availability [39]. Hence, the relocalization of the R2TP complex allows the cells to rapidly respond and control snoRNP biogenesis, and, subsequently, regulate pre-rRNA processing and ribosome biogenesis in response to different growth conditions.

It is interesting to note that Rix7, which is an essential AAA + ATPase required for the biogenesis and nuclear export of $60 \mathrm{~S}$ ribosomal subunits, also undergoes growth phase redistribution [43]. It localizes throughout the 
nucleus in exponentially growing cells, but concentrates in the nucleolus in stationary phase cells. Hence, the dynamic relocalization of protein complexes involved directly or indirectly in ribosome biogenesis might be a generally conserved mechanism designed to allow the cell to easily correlate the number of ribosomes to nutrient availability and growth phase.

It is known that ribosome biogenesis is regulated by the TOR signaling pathway [40], which balances the production of ribosome components to nutrient availability. We observed that the R2TP complex is regulated by the TOR signaling pathway since the localization of the R2TP proteins is affected by the specific inhibitor for TOR, rapamycin. At this stage, we do not know how the TOR pathway effects the nucleo-cytoplasmic translocation of the R2TP complex. However, one intriguing finding from our study points to the presence of a signaling-like pathway that links chaperone activity to ribosome biogenesis, which is based on stabilizing protein complexes (Additional file 6: Figure S6). In our earlier study [14], we had shown that the stability of Pih1 depends on the activity of Hsp90 chaperone together with its co-factor Tah1. This activity then results in the proper assembly of the R2TP complex. In this study, we demonstrated that the proper function and localization of the R2TP complex is required for the stability of Nop58 and assembly of the box C/D snoRNP complexes. The critical players in this pathway are Hsp90, Pih1, and Nop58; both Pih1 and Nop58 are unstable proteins. Importantly, there seems to be a directionality to this signaling-like pathway since functionally deficient Nop58 does not affect Pih1 (Figure 2C), while the destabilization or deletion of Pih1 does affect Nop58 (Figure 2A,B). The presence of such signaling-like pathways that are based on the stabilization of protein complexes rather than protein modification might be widespread in the cell and warrants further investigation.

\section{Conclusions}

Figure 9 provides a summary of our current model of the regulation of box C/D snoRNP biogenesis by the R2TP complex. Most of the R2TP complex localizes in the nucleus in log phase or nutrient rich conditions and associates with the free pool of Nop58 that has not yet assembled with other box C/D snoRNP components. The R2TP-Nop58 interaction stabilizes Nop58, prevents its degradation, and eventually promotes its assembly into the box C/D snoRNP complex. We observed that

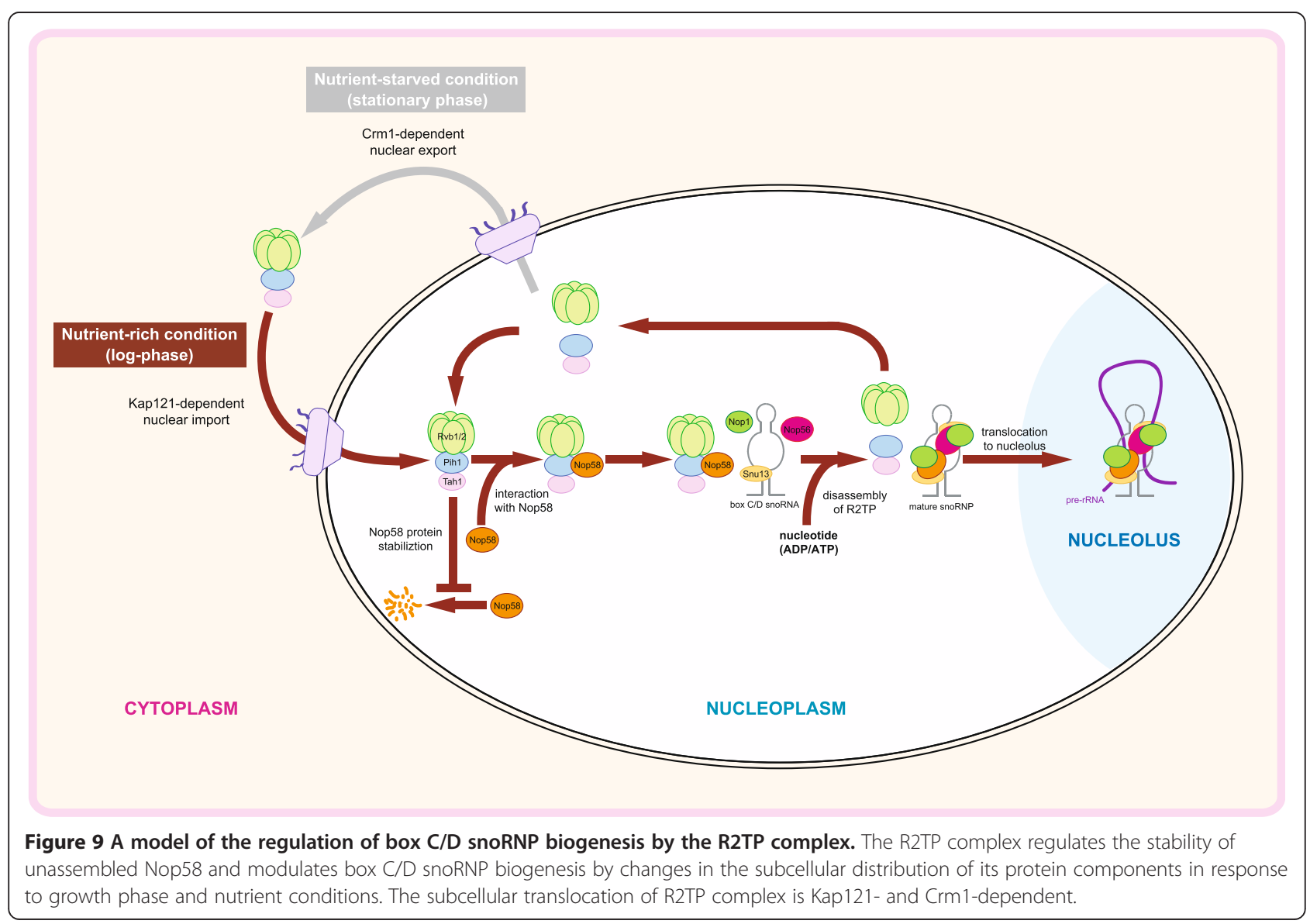


the R2TP complex dissociates from box C/D snoRNPs and that the complex itself disassembles in the presence of nucleotides (Figure 4). Hence, upon nucleotide binding by R2TP, the mature snoRNP then translocates to the nucleolus, while R2TP is recycled. In exponentially growing cells, the R2TP complex is enriched in the nucleus by a Kap121-dependent mechanism to function in the assembly of box C/D snoRNPs. However, in stationary phase or under nutrient limiting conditions, the R2TP complex is actively translocated to the cytoplasm by a Crm1-dependent pathway, and as a result, the assembly of the box C/D snoRNPs is affected, which eventually results in the decrease of ribosome biogenesis. Hence, the regulated subcellular relocalization of R2TP modulates ribosome biogenesis.

\section{Materials and methods \\ Yeast strains and media}

S. cerevisiae strains used in this work are listed in Table 1.

C-terminal 3xFLAG-tagged strains and gene deletion mutants were constructed by PCR-based homologous recombination method [55]. Standard media, YPD, YPGal, and

Table 1 Yeast strains used in this study

\begin{tabular}{|c|c|c|c|}
\hline Name & Background & Genotype & References \\
\hline Y239 & S288C & 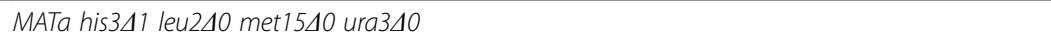 & \\
\hline Y240 & S288C & 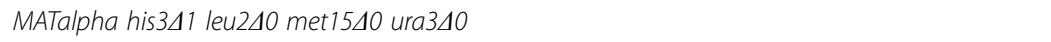 & \\
\hline YK32 & S288C & 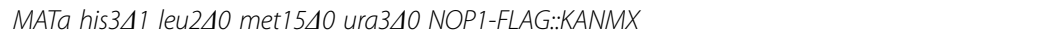 & This study \\
\hline YK68 & S288C & 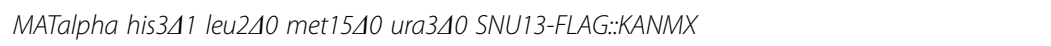 & This study \\
\hline YK3 & S288C & MATa leu2 his3 ura3 lys2 NOP56-FLAG::KANMX & {$[14]$} \\
\hline YK23 & S288C & 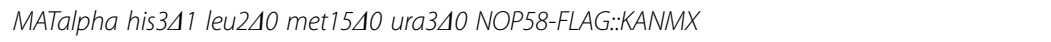 & This study \\
\hline YK28 & S288C & 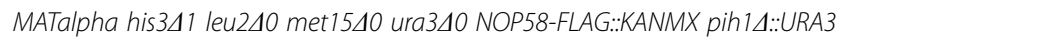 & This study \\
\hline YK29 & S288C & 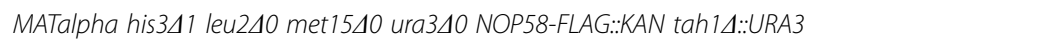 & This study \\
\hline YK26 & S288C & 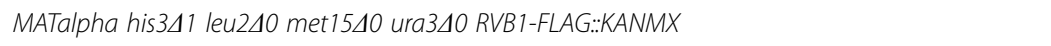 & This study \\
\hline YK27 & S288C & 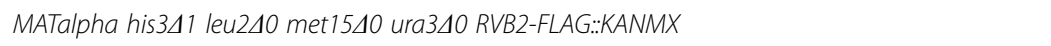 & This study \\
\hline YK31 & S288C & 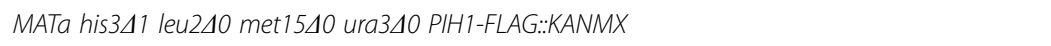 & This study \\
\hline rvb1-DAmP & S288C & MATalpha can1A ::STE2pr-Sp_his5 lyp14 ::STE3pr-LEU2 RVB1-3'UTR::NAT & {$[36]$} \\
\hline rvb2-DAmP & S288C & MATalpha can1A ::STE2pr-Sp_his5 lyp1A ::STE3pr-LEU2 RVB2-3'UTR::NAT & {$[36]$} \\
\hline R0055 & S288C & 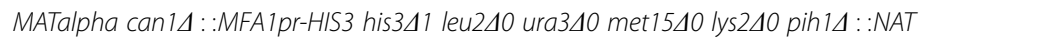 & {$[21]$} \\
\hline R0061 & S288C & 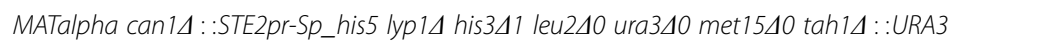 & {$[14]$} \\
\hline nop $58-3^{\prime} \Delta$ & S288C & 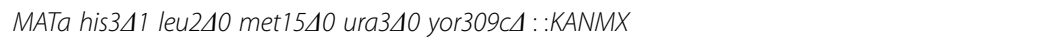 & Open Biosystems \\
\hline RVB1-GFP & S288C & 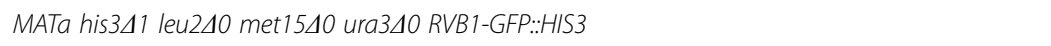 & Life Technologies \\
\hline YK169 & S288C & 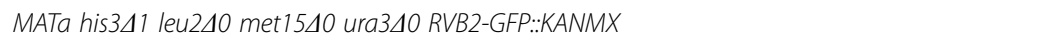 & This study \\
\hline PIH1-GFP & S288C & 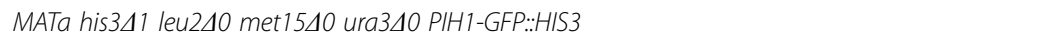 & Life Technologies \\
\hline TAH1-GFP & S288C & 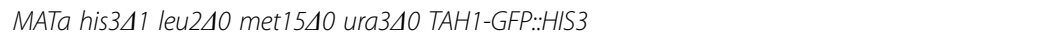 & Life Technologies \\
\hline NOP1-GFP & S288C & 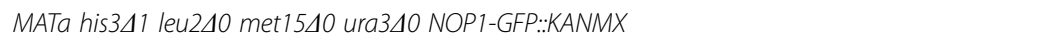 & This study \\
\hline NOP56-GFP & S288C & 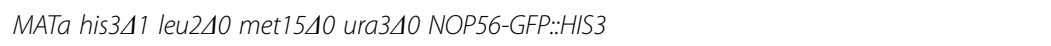 & Life Technologies \\
\hline NOP58-GFP & S288C & 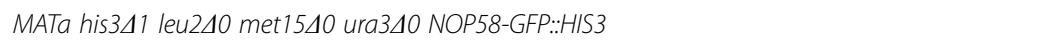 & Life Technologies \\
\hline RVB1-GFP pih1A & S288C & 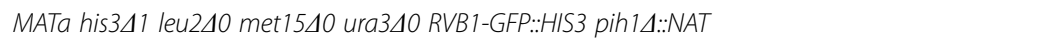 & This study \\
\hline TAH1-GFP pih1A & S288C & 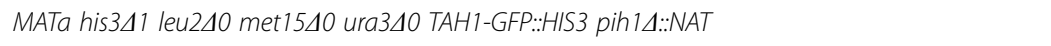 & This study \\
\hline NOP1-GFP pih1A & S288C & 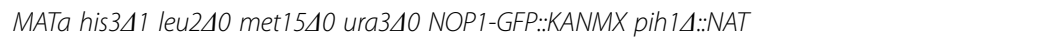 & This study \\
\hline NOP56-GFP pih14 & S288C & 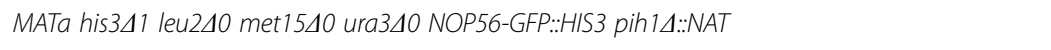 & This study \\
\hline NOP58-GFP pih14 & S288C & 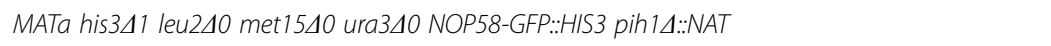 & This study \\
\hline kap121-34 PIH1-GFP & DF5 & MATa ura3-52 his3-4200 trp1-1 leu2-3,112 lys2-801 kap1214::LEU2 pkap121-34-TRP1 PIH1-GFP.:.HIS3 & This study \\
\hline kap121-34 TAH1-GFP & DF5 & MATa ura3-52 his3-4200 trp1-1 leu2-3,112 lys2-801 kap1214::LEU2 pkap121-34-TRP1 TAH1-GFP:.:HIS3 & This study \\
\hline YK124 & S288C & 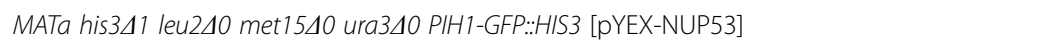 & This study \\
\hline YK125 & S288C & 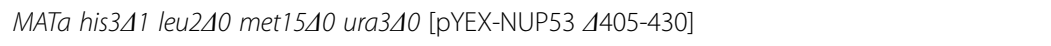 & This study \\
\hline YK175 & S288C & MATa his3 11 leu2 20 ura3A0 crm1-T539C PIH1-GFP.:HIS3 & This study \\
\hline YK176 & S288C & 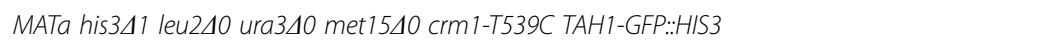 & This study \\
\hline
\end{tabular}


SD containing appropriate supplements were made as described previously [56]. Unless stated otherwise, yeast strains were grown at $30^{\circ} \mathrm{C}$. For rapamycin treatment, log phase cells $\left(\mathrm{OD}_{600}=0.3\right)$ in SD medium were treated with $100 \mathrm{ng} / \mathrm{mL}$ rapamycin for $2 \mathrm{~h}$. For carbon or nitrogen starvation, cells were grown to log phase $\left(\mathrm{OD}_{600}=0.3\right)$ in $\mathrm{SD}$ medium, and washed with distilled water, then incubated in carbon-depleted medium (SD without $2 \%$ dextrose) or nitrogen-depleted medium $(0.17 \%$ yeast nitrogen base without amino acids and ammonium sulfate, and $2 \%$ glucose) for $2 \mathrm{~h}$. For leptomycin B treatment, log phase cells $\left(\mathrm{OD}_{600}=0.3\right)$ were incubated with $20 \mathrm{ng} / \mathrm{mL}$ of leptomycin B for 16 h until stationary phase.

\section{Plasmids}

For expression of GST-tagged Nop58 variants in E. coli, four constructs were prepared, pGST-Nop58-447, pGSTNop58-MC, pGST-Nop58-C, and pGST-Nop58-NM (Figure 1D). The yeast NOP58 gene was amplified by PCR from genomic DNA using the primers listed in Table 2 and then cloned into BamHI-XhoI sites of pGEX-6P-1 vector (GE Healthcare). For overexpression of Nop58-FLAG subdomains for pulldown experiments in yeast, seven constructs were prepared including a negative control, p416GAL-Nop58(1-511)-FLAG, p416GALNop58(285-511)-FLAG, p416GAL-Nop58(345-511)FLAG, p416GAL-Nop58(1-447)-FLAG, p416GAL-Nop58 (285-447)-FLAG, p416GAL-Nop58(345-447)-FLAG, and p416GAL-GFP-FLAG. For p416GAL-Nop58(1-511)FLAG, p416GAL-Nop58(285-511)-FLAG, and p416GALNop58(345-511)-FLAG, the FLAG-tagged NOP58 DNA fragments were amplified from genomic DNA of NOP58FLAG strain by PCR using primers listed in Table 2 and inserted into BamHI-XhoI sites of p416GAL. For p416GAL-Nop58(1-447)-FLAG, p416GAL-Nop58(285447)-FLAG, and p416GAL-Nop58(345-447)-FLAG constructs, the NOP58 DNA fragments were amplified from wildtype yeast genomic DNA using primers listed in Table 2. The GFP DNA sequence was amplified by PCR from pFA6a-GFP(S65T)-KanMX plasmid using primers in Table 2 and cloned into BamHI-XhoI sites of p416GAL vector.

For p416GAL-PIH1 (Figure 2B), the yeast PIH1 gene was PCR amplified from genomic DNA using oligonucleotides (Table 2) and cloned into BamHI-XhoI sites of p416GAL vector. The pYEX-NUP53 and pYEXNUP53-C $\Delta$ plasmids were a kind gift from Dr. Richard W. Wozniak (University of Alberta). To influence the localization of Pih1-GFP four plasmids were constructed: pRS315-PIH1-GFP-NLS, pRS315-PIH1-GFP-mNLS, pRS315-PIH1-GFP-NES, and pRS315-PIH1-GFP-mNES. The DNA sequences were amplified from genomic DNA isolated from PIH1-GFP genomically-tagged strain.

\section{Protein purification}

The construction, expression, and purification of fulllength Pih1 and Pih1(1-230) have been described previously $[14,26]$. The Tah1 and Pih1 proteins were co-expressed using co-expression vector, pCOLADuet-1 transformed into BL21(DE3) pRIL cells. Tah1 had an Nterminal $\mathrm{His}_{6}$-tag followed by tobacco etch virus cut site (TEV), HV-tag, while Pih1 was untagged. Protein expression was induced with $1 \mathrm{mM}$ IPTG at $18^{\circ} \mathrm{C}$. The co-expressed HV-Tah1/Pih1 complex was purified using Ni-NTA resin, and, subsequently, the N-terminal HV-Tag on Tah1 was removed by incubating with TEV protease. The Tah1-Pih1 complex was further purified on an anion exchange chromatography column (Mono Q). HVRvb1 and untagged Rvb2 were also co-expressed using pCOLADuet-1 in BL21(DE3) pRIL, and purified using NiNTA resin column. The N-terminal HV-tag of Rvb1 was removed by TEV protease as described above. Subsequently, the Rvb1/Rvb2 complex was purified on Superdex 200 size exclusion column.

\section{FLAG pulldown assays}

FLAG pulldown assays were performed essentially as described [14,57]. C-terminal 3xFLAG-tagged strains were grown in YPD medium to log phase $\left(\mathrm{OD}_{600}=\right.$ approximately 0.6$)$ or stationary phase $\left(\mathrm{OD}_{600}>5.0\right)$ at $30^{\circ} \mathrm{C}$. For Figure $1 \mathrm{~B}$, soluble cell lysate was treated with DNase I $(100 \mathrm{U} / \mathrm{mL})$ and/or RNase $\mathrm{A}(0.5 \mathrm{mg} / \mathrm{mL})$ at $30^{\circ} \mathrm{C}$ for 30 min before incubating with anti-FLAG (M2) sepharose beads (Sigma). For Figure 1E, the transformants of each p416GAL construct were grown on SD-Ura plates for 2 days at $30^{\circ} \mathrm{C}$ and incubated in SG (galactose)-Ura medium until $\log$ phase for $6 \mathrm{~h}$ at $30^{\circ} \mathrm{C}$. Cell pellets were disrupted with dry ice using a coffee grinder and dissolved in buffer $\mathrm{H}$ [25 mM HEPES-KOH, pH 7.6, $1 \mathrm{mM}$ EDTA, 10\% glycerol, 0.02\% NP-40, 2.5 mM DTT, 2 mM $\mathrm{MgCl}_{2}$, and protease inhibitor cocktail tablets (Roche Diagnostics)] containing $0.1 \mathrm{M} \mathrm{KCl}$. The total cell lysate was clarified by centrifugation and then the supernatant was used for immunoprecipitation using anti-FLAG beads. The immunopurified protein complexes were washed with buffer $\mathrm{H}$ containing $0.3 \mathrm{M}$ or $0.5 \mathrm{M} \mathrm{KCl}$. $1 \mathrm{mg} / \mathrm{mL}$ FLAG peptide (Sigma) in $0.1 \mathrm{M} \mathrm{KCl}$ was used for elution. For Figure 4, the complexes on the $\alpha$-FLAG beads were further incubated in the absence or presence of $4 \mathrm{mM}$ nucleotides at $30^{\circ} \mathrm{C}$ for $30 \mathrm{~min}$, and the supernatants were removed. The beads were washed with buffer $\mathrm{H}$ containing $0.1 \mathrm{M} \mathrm{KCl}$. The levels of the proteins in each fraction were determined by Western blotting or silver staining.

\section{GST pulldown assays}

Nop58 protein fragments with N-terminal GST-tag were expressed in E. coli BL21(DE3) pRIL cells. The fusion 
Table 2 Primers used in this study for PCR

\begin{tabular}{|c|c|c|}
\hline Plasmid/Gene names & Primers & Sequences \\
\hline \multirow[t]{2}{*}{ pGEX-Nop58-447 } & NOP58-BHI-F & TTGGATCCATGGCTTACGTITIAACTGAAACTTC \\
\hline & NOP58-447-Xhol-R & TTTCTCGAGTTATTCATCATCAGAATCGGATTCAGAATC \\
\hline \multirow[t]{2}{*}{ pGEX-Nop58-MC } & NOP58-472-BamHI-F & TTGGATCCATGAAAGTTGATGTTATGATTATTCAAGCA \\
\hline & NOP58-447-Xhol-R & TTTCTCGAGTTATTCATCATCAGAATCGGATTCAGAATC \\
\hline \multirow[t]{2}{*}{ pGEX-Nop58-C } & NOP58-853-BamHI-F & TTGGATCCATGCCAAACTTGACTCAGTTGGTTG \\
\hline & NOP58-447-Xhol-R & TTTCTCGAGTTATTCATCATCAGAATCGGATTCAGAATC \\
\hline \multirow[t]{2}{*}{ pGEX-Nop58-NM } & NOP58-BHI-F & TTGGATCCATGGCTTACGTITTAACTGAAACTTC \\
\hline & NOP58-852-Xhol-R & TTTCTCGAGTTAAGCAATAGCCTTCATTCTTGCAG \\
\hline \multirow[t]{2}{*}{ p416GAL-GFP-FLAG } & GFPs65t-BamHI-F & TTGGATCCATGAGTAAAGGAGAAGAACTITCACTGGAG \\
\hline & GFP-nonstop-Xhol-R & TTTCTCGAGTTTGTATAGTTCATCCATGCCATG \\
\hline \multirow[t]{2}{*}{ p416GAL-Nop58(1-511)-FLAG } & NOP58-BHI-F & TTGGATCCATGGCTTACGTITTAACTGAAACTTC \\
\hline & FLAG-Xhol-R & TTTCTCGAGCTATTTATCGTCATCATCTTTGTAGTCCTTGTC \\
\hline \multirow[t]{2}{*}{ p416GAL-Nop58(285-511)-FLAG } & NOP58-853-BamHI-F2 & TTGGATCCATGCCAAACTTGACTCAGTTGGTTGGTGAAT \\
\hline & FLAG-Xhol-R & TTCTCGAGCTATTTATCGTCATCATCTTTGTAGTCCTTGTC \\
\hline \multirow[t]{2}{*}{ p416GAL-Nop58(345-511)-FLAG } & NOP58-1033-BamHI-F & TTGGATCCATGGCCTCTCTTGTTGGTCAAGCTACTGGT \\
\hline & FLAG-Xhol-R & TTTCTCGAGCTATTTATCGTCATCATCTTTGTAGTCCTTGTC \\
\hline \multirow[t]{2}{*}{ p416GAL-Nop58(1-447)-FLAG } & NOP58-BHI-F & TTGGATCCATGGCTTACGTITIAACTGAAACTTC \\
\hline & NOP58-447-nostop-Xhol-R & TTTCTCGAGTTCATCATCAGAATCGGATTCAGAATC \\
\hline \multirow[t]{2}{*}{ p416GAL-Nop58(285-447)-FLAG } & NOP58-853-BamHI-F & TTGGATCCATGCCAAACTTGACTCAGTTGGTTG \\
\hline & NOP58-447-nostop-Xhol-R & TTTCTCGAGTTCATCATCAGAATCGGATTCAGAATC \\
\hline \multirow[t]{2}{*}{ p416GAL-Nop58(345-447)-FLAG } & NOP58-1033-BamHI-F & TTGGATCCATGGCCTCTCTTGTTGGTCAAGCTACTGGT \\
\hline & NOP58-447-nostop-Xhol-R & TTTCTCGAGTTCATCATCAGAATCGGATTCAGAATC \\
\hline \multirow[t]{2}{*}{ FLAG } & FLAG-Xhol-F & TTTCTCGAGATGGATTACAAGGATGACGACGAT \\
\hline & FLAG-Xhol-noPae-R & TTTCTCGAGAGCTATTTATCGTCATCATCTTTGTAGTCCTTGTC \\
\hline \multirow[t]{2}{*}{ p416GAL-PIH1 } & $\mathrm{PIH1}-\mathrm{BHI}-\mathrm{F}$ & TTGGATCCATGGCCGATTTCTTATTGAGACC \\
\hline & $\mathrm{PlH} 1-\mathrm{Xhol}-\mathrm{R}$ & TTCTCGAGTTATATATATATATATAGTGTGCGT \\
\hline \multirow[t]{2}{*}{ pRS315-PIH1-GFP-NLS } & PIH1-m413-Xbal-F & AAATCTAGAGGAAGGTACCAGTGGCAATTCAGCA \\
\hline & GFP-NLS-Xhol-R & $\begin{array}{l}\text { TाTCTCGAGTTAAACTTIACGTITCTTCTITGGAGACTCTTCT } \\
\text { TCGGAACCATCAGCAGTACGCTIITGTATAGTTCATCCAT } \\
\text { GCCATGTGTAATCCCAG }\end{array}$ \\
\hline \multirow[t]{2}{*}{ pRS315-PIH1-GFP-mNLS } & PIH1-m413-Xbal-F & AAATCTAGAGGAAGGTACCAGTGGCAATTCAGCA \\
\hline & GFP-mNLS-Xhol-R & $\begin{array}{l}\text { TTCTCGAGTTAAACTTIACGTTAGCCTTGGAGACTCTTC } \\
\text { TTCGGAACCATCAGCAGTGGCTGCTITGTATAGTTCATCCA } \\
\text { TGCCATGTGTAATCCCAG }\end{array}$ \\
\hline \multirow[t]{2}{*}{ pRS315-PIH1-GFP-NES } & PIH1-m413-Xbal-F & AAATCTAGAGGAAGGTACCAGTGGCAATTCAGCA \\
\hline & GFP-NES-Xhol-R & $\begin{array}{l}\text { TITCTCGAGTTAAGTCTTATTGATGTCTAAACCAGCTAACTT } \\
\text { CAAAGCTAAACCTGGAGCTTTGTATAGTTCATCCATGCCAT } \\
\text { GTGTAATCCCAG }\end{array}$ \\
\hline \multirow[t]{2}{*}{ pRS315-PIH1-GFP-mNES } & $\mathrm{PIH1}-\mathrm{m} 413-\mathrm{Xbal}-\mathrm{F}$ & AAATCTAGAGGAAGGTACCAGTGGCAATTCAGCA \\
\hline & GFP-mNES-Xhol-R & $\begin{array}{l}\text { TTCTCGAGTTAAGTCTTATTAGTGTCAGCACCAGCTAACTT } \\
\text { CAAAGCTAAACCTGGAGCTITGTATAGTTCATCCATGCCAT } \\
\text { GTGTAATCCCAG }\end{array}$ \\
\hline
\end{tabular}

proteins were purified on glutathione Sepharose 4B beads following manufacturer's protocol (GE Healthcare). Pih1 and Pih1(1-230) were purified using the same protocol as previously described [14]. The immobilized GST-Nop58 variants were incubated with Pih1 or Pih1(1-230) for
$40 \mathrm{~min}$ at $30^{\circ} \mathrm{C}$ in GST lysis buffer $(1 \times \mathrm{PBS}, 0.1 \% \mathrm{NP} 40$, $10 \%$ glycerol, and $1 \mathrm{mM}$ DTT). The beads were washed three times with GST lysis buffer containing $0.5 \mathrm{M} \mathrm{NaCl}$ and then rinsed with the GST lysis buffer. The retained proteins were separated on SDS-PAGE gels and visualized 
by Ponceau S staining and Western blotting. For in vitro reconstitution of Nop58 C-terminal domain with R2TP complex (Figure 4D), MagneGST Glutathione Particles (Promega) were used for purification of GST alone and GST-Nop58-C proteins (Figure 1D, upper panel). Then, $0.1 \mu \mathrm{M}$ of preformed Rvb1/2 or R2TP complex purified from $E$. coli was incubated with the purified glutathione beads-bound proteins at $4^{\circ} \mathrm{C}$ for $16 \mathrm{~h}$, and the unbound R2TP was washed away using buffer $\mathrm{H}$ containing $0.1 \mathrm{M}$ $\mathrm{KCl}$ at $23^{\circ} \mathrm{C}$.

\section{Northern blot analysis}

Total RNA extraction and Northern analysis were performed as described [14]. Briefly, total RNA from log phase cells and co-immunoprecipitated RNA from FLAG pulldowns were extracted by phenol/chloroform extraction and precipitated by ethanol with $0.5 \mathrm{M} \mathrm{LiCl}$. For snoRNA Northern analysis, $6 \mu \mathrm{g}$ and $0.6 \mu \mathrm{g}$ of total RNA, and RNA isolated from pulldown complexes were separated on 5\% polyacrylamide-urea gels and electrotransferred to a Biodyne B membrane (Pall). The transferred membrane was hybridized using $5{ }^{\prime}-{ }^{32} \mathrm{P}$-labeled oligonucleotide probes for U14 (GCGGTCACCGAGAGTAC TAACGA) (Figure 3A).

\section{RT-PCR}

FLAG pulldown complexes eluted with 3x FLAG peptide and input soluble cell lysates for the pulldown were treated with RNase-free DNase I at $37^{\circ} \mathrm{C}$ for $30 \mathrm{~min}$ and then the RNAs were extracted with phenol/chloroform and precipitated by ethanol with $0.5 \mathrm{M} \mathrm{LiCl}$. The first strand cDNA was synthesized using reverse primer for U14, U14-90-R (CGGTCACCGAGAGTAC TAACG), dNTP, and reverse transcriptase (SuperScript ${ }^{\mathrm{tm}}$ III, Life Technologies) from the purified RNA template. The synthesized cDNAs were amplified by PCR using U14-m60-F (TCTCATGAGATTATCAAATGTGGG) and U14-90R as a primer set to detect U14 pre-snoRNA.

\section{Western blot analysis}

For the analysis of protein accumulation in cells, total crude cell lysate was prepared as previously reported [58]. Proteins resolved by SDS-PAGE were transferred to nitrocellulose membranes (Pall). The membrane blots were blocked with $5 \%$ non-fat dried milk and incubated for $2 \mathrm{~h}$ or overnight with antibodies. The antibodies used were as follows: Rabbit $\alpha$-Rvb1, $\alpha$-Rvb2, $\alpha$-Pih1, and $\alpha$ Tah1 [14], mouse monoclonal $\alpha$-FLAG (Sigma), $\alpha$-actin (Abcam), $\alpha$-Nop1 (clone 28 F2, EnCor biotechnology Inc.), and $\alpha$-Nop58 (clone 34B12, EnCor biotechnology Inc.). Polyclonal rabbit antisera for Snu13 and Nop56 were produced using purified recombinant Snu13 and Nop56 proteins from E. coli (EZBiolab Inc.). Goat $\alpha-$ rabbit or goat $\alpha$-mouse IgG antibodies conjugated with horseradish peroxidise (Biorad) were used as secondary antibodies.

\section{Microscopy}

Cells were mounted on glass slides for image analysis. Images were captured using a DMI 6000B fluorescence microscope (Leica Microsystems) at $63 \times$ magnification equipped with a spinning-disk head, an argon laser (Quorum Technologies) and ImagEM charge-coupled device camera (Hamamatsu Photonics). For nuclear staining of live cells, yeast cultures were incubated for 10 min with $6 \times$ diluted NucBlue Live Cell Stain (Hoechst33342; Life Technologies) before capturing the image. For quantification of GFP signals in the nucleolus and nucleoplasm shown in Figure 6D, the densitometry of GFP signals of approximately 100 cells were measured using NIH ImageJ. The total GFP signals were measured and set as $100 \%$, and the GFP signals which did not overlap with an area stained by Hoechst 33342 were counted as nucleolus. For nuclear import analysis of Figure 7B, the transformant of pYEX-NUP53-C $\Delta$ or pYEX-NUP53 was grown in SD-Ura until early-log phase, and then washed with SD-Leu-Ura medium and further incubated in SD-Leu-Ura medium for $3 \mathrm{~h}$.

\section{Additional files}

Additional file 1: Figure S1. Wider view of the subcellular localizations of endogenously GFP-tagged R2TP and box C/D snoRNP proteins in log and stationary phase cells. Cells were stained with Hoechst33342 and then analyzed for GFP or Hoechst33342 fluorescence. The images of DIC, GFP (green), and Hoechst33342 (red), and the merged pictures are shown. White arrowheads show nucleoplasmic GFP signals of Nop1-, Nop56-, and Nop58-GFP.

Additional file 2: Figure S2. Wider view of the subcellular localizations of endogenously GFP-tagged Rvb1 and Tah1 in WT and pih1 1 log phase cells. Cells were stained with Hoechst33342 and then analyzed for GFP or Hoechst33342 fluorescence. The images of DIC, GFP (green), and Hoechst33342 (red), and the merged pictures are shown.

Additional file 3: Figure S3. Wider view of the subcellular localizations of endogenously GFP-tagged Nop1, Nop56, and Nop58 in WT and pih14 log phase cells. Cells were stained with Hoechst33342 and then analyzed for GFP or Hoechst33342 fluorescence. The images of DIC, GFP (green), and Hoechst33342 (red), and the merged pictures are shown. White arrowheads show nucleoplasmic GFP signals of Nop1-GFP, Nop56-GFP, and Nop58-GFP.

Additional file 4: Figure S4. Subcellular localizations in log phase cells of endogenously GFP-tagged R2TP and box C/D proteins in SD media, nutrient-limited media lacking carbon or nitrogen sources, and after rapamycin treatment. The images of DIC and GFP signals from GFP-tagged R2TP and box C/D snoRNP proteins are shown.

Additional file 5: Figure S5. Subcellular localizations of endogenously GFP-tagged Rvb1 and Rvb2 in log phase WT cells expressing Nup53-C $\Delta$ or Nup53 under CUP1 promoter. The images of DIC and GFP signals from GFP-tagged Rvb1 and Rvb2 are shown.

Additional file 6: Figure S6. A model of a signaling-like pathway based on protein stabilization affecting ribosome biogenesis. The unstable protein Pih1 is stabilized by the Hsp90-Tah1 chaperone complex, while Nop58 is stabilized by the R2TP-Hsp90 complex. This protein stabilization pathway regulates box C/D snoRNP maturation and, hence, ribosome biogenesis. 


\section{Competing interests}

The authors declare that they have no competing interests.

\section{Authors' contributions}

YK and WAH designed the study. YK designed and performed most of the experiments. TM helped with microscopy. LZ helped with protein purification and WT helped with some of the pulldown assays. YK and WAH wrote the manuscript. All authors read and approved the final manuscript.

\section{Acknowledgements}

We thank Dr. Charles Boone (University of Toronto), Dr. Brenda Andrew (University of Toronto), Dr. Timothy Hughes (University of Toronto), Dr. Richard W. Wozniak (University of Alberta), Dr. Shunichi Kosugi (Kazusa DNA Research Institute), and Dr. Hiroshi Yanagawa (Keio University) for the kind gift of yeast strains. We also thank Dr. Richard W. Wozniak (University of Alberta) and Dr. Karsten Weis (University of California, Berkeley) for providing antibodies for Kap121 and Crm1, respectively. YK is a postdoctoral fellow of the Canadian Institutes of Health Research (ClHR) Training Program Grant in Protein Folding: Principles and Diseases (TGF-53910). This work was funded by a CIHR grant (MOP-93778) to WAH.

Received: 23 April 2014 Accepted: 25 July 2014

Published: 25 July 2014

\section{References}

1. Venema J, Tollervey D: Ribosome synthesis in Saccharomyces cerevisiae. Annu Rev Genet 1999, 33:261-311.

2. Warner JR: The economics of ribosome biosynthesis in yeast. Trends Biochem Sci 1999, 24:437-440.

3. Kressler D, Doere M, Rojo M, Linder P: Synthetic lethality with conditional dbp6 alleles identifies rsa1p, a nucleoplasmic protein involved in the assembly of 60S ribosomal subunits. Mol Cell Biol 1999, 19:8633-8645.

4. Butler JS: The yin and yang of the exosome. Trends Cell Biol 2002, 12:90-96.

5. Cordin O, Banroques J, Tanner NK, Linder P: The DEAD-box protein family of RNA helicases. Gene 2006, 367:17-37.

6. Bertrand E, Bordonne R: Assembly and traffic of small nuclear RNPs. Prog Mol Subcell Biol 2004, 35:79-97.

7. Cavaille J, Nicoloso M, Bachellerie JP: Targeted ribose methylation of RNA in vivo directed by tailored antisense RNA guides. Nature 1996, 383:732-735.

8. Kiss-Laszlo Z, Henry Y, Kiss T: Sequence and structural elements of methylation guide snoRNAs essential for site-specific ribose methylation of pre-rRNA. EMBO J 1998, 17:797-807.

9. Tollervey D, Lehtonen $H$, Jansen $R$, Kern H, Hurt EC: Temperature-sensitive mutations demonstrate roles for yeast fibrillarin in pre-rRNA processing, pre-rRNA methylation, and ribosome assembly. Cell 1993, 72:443-457.

10. Omer AD, Ziesche $S$, Ebhardt $H$, Dennis PP: In vitro reconstitution and activity of a C/D box methylation guide ribonucleoprotein complex. Proc Natl Acad Sci U S A 2002, 99:5289-5294.

11. Watkins NJ, Dickmanns A, Luhrmann R: Conserved stem II of the box C/D motif is essential for nucleolar localization and is required, along with the $15.5 \mathrm{~K}$ protein, for the hierarchical assembly of the box C/D snoRNP. Mol Cell Biol 2002, 22:8342-8352.

12. Yang Y, Isaac C, Wang C, Dragon F, Pogacic V, Meier UT: Conserved composition of mammalian box H/ACA and box C/D small nucleolar ribonucleoprotein particles and their interaction with the common factor Nopp140. Mol Biol Cell 2000, 11:567-577.

13. Boulon S, Marmier-Gourrier N, Pradet-Balade B, Wurth $L$, Verheggen C, Jady BE, Rothe B, Pescia C, Robert MC, Kiss T, Bardoni B, Krol A, Branlant C, Allmang C, Bertrand E, Charpentier B: The Hsp90 chaperone controls the biogenesis of L7Ae RNPs through conserved machinery. J Cell Biol 2008, 180:579-595.

14. Zhao R, Kakihara Y, Gribun A, Huen J, Yang G, Khanna M, Costanzo M, Brost RL, Boone C, Hughes TR, Yip CM, Houry WA: Molecular chaperone Hsp90 stabilizes Pih1/Nop17 to maintain R2TP complex activity that regulates snoRNA accumulation. J Cell Biol 2008, 180:563-578.

15. Peng WT, Robinson MD, Mnaimneh S, Krogan NJ, Cagney G, Morris Q, Davierwala AP, Grigull J, Yang X, Zhang W, Mitsakakis N, Ryan OW, Datta N, Jojic V, Pal C, Canadien V, Richards D, Beattie B, Wu LF, Altschuler SJ, Roweis
S, Frey BJ, Emili A, Greenblatt JF, Hughes TR: A panoramic view of yeast noncoding RNA processing. Cell 2003, 113:919-933.

16. Boulon S, Verheggen C, Jady BE, Girard C, Pescia C, Paul C, Ospina JK, Kiss T, Matera AG, Bordonne R, Bertrand E: PHAX and CRM1 are required sequentially to transport U3 snoRNA to nucleoli. Mol Cell 2004, 16:777-787.

17. Watkins NJ, Lemm I, Ingelfinger D, Schneider C, Hossbach M, Urlaub H, Luhrmann R: Assembly and maturation of the U3 snoRNP in the nucleoplasm in a large dynamic multiprotein complex. Mol Cell 2004, 16:789-798.

18. McKeegan KS, Debieux CM, Boulon S, Bertrand E, Watkins NJ: A dynamic scaffold of pre-snoRNP factors facilitates human box C/D snoRNP assembly. Mol Cell Biol 2007, 27:6782-6793.

19. Watkins NJ, Lemm I, Luhrmann R: Involvement of nuclear import and export factors in U8 box C/D snoRNP biogenesis. Mol Cell Biol 2007, 27:7018-7027.

20. Pradet-Balade B, Girard C, Boulon S, Paul C, Azzag K, Bordonne R, Bertrand E, Verheggen C: CRM1 controls the composition of nucleoplasmic presnoRNA complexes to licence them for nucleolar transport. EMBO J 2011, 30:2205-2218

21. Zhao R, Davey M, Hsu YC, Kaplanek P, Tong A, Parsons AB, Krogan N, Cagney G, Mai D, Greenblatt J, Boone C, Emili A, Houry WA: Navigating the chaperone network: an integrative map of physical and genetic interactions mediated by the hsp90 chaperone. Cell 2005, 120:715-727.

22. Kakihara $Y$, Houry WA: The R2TP complex: discovery and functions. Biochim Biophys Acta 2012, 1823:101-107.

23. Jha S, Dutta A: RVB1/RVB2: running rings around molecular biology. Mol Cell 2009, 34:521-533.

24. Huen J, Kakihara Y, Ugwu F, Cheung KL, Ortega J, Houry WA: Rvb1-Rvb2: essential ATP-dependent helicases for critical complexes. Biochem Cell Biol 2010, 88:29-40.

25. Gonzales FA, Zanchin NI, Luz JS, Oliveira CC: Characterization of Saccharomyces cerevisiae Nop17p, a novel Nop58p-interacting protein that is involved in Pre-rRNA processing. J Mol Biol 2005, 346:437-455.

26. Jimenez B, Ugwu F, Zhao R, Orti L, Makhnevych T, Pineda-Lucena A, Houry WA: Structure of minimal tetratricopeptide repeat domain protein Tah1 reveals mechanism of its interaction with Pih1 and $\mathrm{Hsp} 90$. J Biol Chem 2012, 287:5698-5709.

27. Jeronimo C, Forget D, Bouchard A, Li Q, Chua G, Poitras C, Therien C, Bergeron D, Bourassa S, Greenblatt J, Chabot B, Poirier GG, Hughes TR, Blanchette M, Price DH, Coulombe B: Systematic analysis of the protein interaction network for the human transcription machinery reveals the identity of the 7SK capping enzyme. Mol Cell 2007, 27:262-274.

28. Boulon S, Pradet-Balade B, Verheggen C, Molle D, Boireau S, Georgieva M, Azzag K, Robert MC, Ahmad Y, Neel H, Lamond Al, Bertrand E: HSP90 and its R2TP/Prefoldin-like cochaperone are involved in the cytoplasmic assembly of RNA polymerase II. Mol Cell 2010, 39:912-924.

29. Horejsi Z, Takai H, Adelman CA, Collis SJ, Flynn H, Maslen S, Skehel JM, de Lange T, Boulton SJ: CK2 phospho-dependent binding of R2TP complex to TEL2 is essential for MTOR and SMG1 stability. Mol Cell 2010, 39:839-850.

30. Inoue M, Saeki M, Egusa H, Niwa H, Kamisaki Y: PIH1D1, a subunit of R2TP complex, inhibits doxorubicin-induced apoptosis. Biochem Biophys Res Commun 2010, 403:340-344.

31. Kamano Y, Saeki M, Egusa H, Kakihara Y, Houry WA, Yatani H, Kamisaki Y: PIH1D1 interacts with mTOR complex 1 and enhances ribosome RNA transcription. FEBS Lett 2013, 587:3303-3308.

32. Saeki M, Egusa H, Kamano Y, Kakihara Y, Houry WA, Yatani H, Noguchi S, Kamisaki Y: Exosome-bound WD repeat protein Monad inhibits breast cancer cell invasion by degrading amphiregulin mRNA. PLoS One 2013, 8:e67326.

33. Ye K, Jia R, Lin J, Ju M, Peng J, Xu A, Zhang L: Structural organization of box C/D RNA-guided RNA methyltransferase. Proc Natl Acad Sci U S A 2009, 106:13808-13813.

34. Lin J, Lai S, Jia R, Xu A, Zhang L, Lu J, Ye K: Structural basis for site-specific ribose methylation by box C/D RNA protein complexes. Nature 2011, 469:559-563.

35. Paci A, Liu XH, Huang H, Lim A, Houry WA, Zhao R: The stability of the small nucleolar ribonucleoprotein (snoRNP) assembly protein Pih1 in Saccharomyces cerevisiae is modulated by its $\mathrm{C}$ terminus. J Biol Chem 2012, 287:43205-43214. 
36. Schuldiner M, Collins SR, Thompson NJ, Denic V, Bhamidipati A, Punna T, Ihmels J, Andrews B, Boone C, Greenblatt JF, Weissman JS, Krogan NJ: Exploration of the function and organization of the yeast early secretory pathway through an epistatic miniarray profile. Cell 2005, 123:507-519.

37. Gribun A, Cheung KL, Huen J, Ortega J, Houry WA: Yeast Rvb1 and Rvb2 are ATP-dependent DNA helicases that form a heterohexameric complex. J Mol Biol 2008, 376:1320-1333.

38. King TH, Decatur WA, Bertrand E, Maxwell ES, Fournier MJ: A wellconnected and conserved nucleoplasmic helicase is required for production of box C/D and H/ACA snoRNAs and localization of snoRNP proteins. Mol Cell Biol 2001, 21:7731-7746.

39. Zaman S, Lippman SI, Zhao X, Broach JR: How Saccharomyces responds to nutrients. Annu Rev Genet 2008, 42:27-81.

40. Rohde JR, Bastidas R, Puria R, Cardenas ME: Nutritional control via Tor signaling in Saccharomyces cerevisiae. Curr Opin Microbiol 2008, 11:153-160.

41. Rout MP, Blobel G, Aitchison JD: A distinct nuclear import pathway used by ribosomal proteins. Cell 1997, 89:715-725.

42. Makhnevych T, Lusk CP, Anderson AM, Aitchison JD, Wozniak RW: Cell cycle regulated transport controlled by alterations in the nuclear pore complex. Cell 2003, 115:813-823.

43. Gadal O, Strauss D, Kessl J, Trumpower B, Tollervey D, Hurt E: Nuclear export of 60 s ribosomal subunits depends on Xpo1p and requires a nuclear export sequence-containing factor, Nmd3p, that associates with the large subunit protein Rpl10p. Mol Cell Biol 2001, 21:3405-3415

44. Johnson AW, Lund E, Dahlberg J: Nuclear export of ribosomal subunits. Trends Biochem Sci 2002, 27:580-585.

45. Neville M, Rosbash M: The NES-Crm1p export pathway is not a major mRNA export route in Saccharomyces cerevisiae. EMBO J 1999, 18:3746-3756

46. Arevalo-Rodriguez M, Heitman J: Cyclophilin A is localized to the nucleus and controls meiosis in Saccharomyces cerevisiae. Eukaryot Cell 2005, 4:17-29.

47. Gautier T, Berges T, Tollervey D, Hurt E: Nucleolar KKE/D repeat proteins Nop56p and Nop58p interact with Nop1p and are required for ribosome biogenesis. Mol Cell Biol 1997, 17:7088-7098.

48. Lafontaine $\mathrm{DL}$, Tollervey D: Synthesis and assembly of the box $C+D$ small nucleolar RNPs. Mol Cell Biol 2000, 20:2650-2659.

49. Colau G, Thiry M, Leduc V, Bordonne R, Lafontaine DL: The small nucle(ol) ar RNA cap trimethyltransferase is required for ribosome synthesis and intact nucleolar morphology. Mol Cell Biol 2004, 24:7976-7986.

50. Mouaikel J, Verheggen C, Bertrand E, Tazi J, Bordonne R: Hypermethylation of the cap structure of both yeast snRNAs and snoRNAs requires a conserved methyltransferase that is localized to the nucleolus. Mol Cell 2002, 9:891-901.

51. Rothe B, Back R, Quinternet M, Bizarro J, Robert MC, Blaud M, Romier C, Manival X, Charpentier B, Bertrand E, Branlant C: Characterization of the interaction between protein Snu13p/15.5 K and the Rsa1p/NUFIP factor and demonstration of its functional importance for snoRNP assembly. Nucleic Acids Res 2014, 42:2015-2036.

52. Stavreva DA, Kawasaki M, Dundr M, Koberna K, Muller WG, TsujimuraTakahashi T, Komatsu W, Hayano T, Isobe T, Raska I, Misteli T, Takahashi N, McNally JG: Potential roles for ubiquitin and the proteasome during ribosome biogenesis. Mol Cell Biol 2006, 26:5131-5145.

53. Matic I, Schimmel J, Hendriks IA, van Santen MA, van de Rijke F, van Dam H, Gnad F, Mann M, Vertegaal AC: Site-specific identification of SUMO-2 targets in cells reveals an inverted SUMOylation motif and a hydrophobic cluster SUMOylation motif. Mol Cell 2010, 39:641-652.

54. Westman BJ, Verheggen C, Hutten S, Lam YW, Bertrand E, Lamond Al: A proteomic screen for nucleolar SUMO targets shows SUMOylation modulates the function of Nop5/Nop58. Mol Cell 2010, 39:618-631.

55. Longtine MS, McKenzie A 3rd, Demarini DJ, Shah NG, Wach A, Brachat A Philippsen P, Pringle JR: Additional modules for versatile and economical PCR-based gene deletion and modification in Saccharomyces cerevisiae. Yeast 1998, 14:953-961.
56. Burke D, Dawson D, Stearns T: Methods in Yeast Genetics. Cold Spring Harbor, NY: Cold Spring Harbor Laboratory; 2000.

57. Shen X: Preparation and analysis of the INO80 complex. Methods Enzymol 2004, 377:401-412.

58. Kushnirov $\mathrm{W}$ : Rapid and reliable protein extraction from yeast. Yeast 2000, 16:857-860

doi:10.1186/s13059-014-0404-4

Cite this article as: Kakihara et al:: Nutritional status modulates box C/D snoRNP biogenesis by regulated subcellular relocalization of the R2TP complex. Genome Biology 2014 15:404.

\section{Submit your next manuscript to BioMed Central and take full advantage of:}

- Convenient online submission

- Thorough peer review

- No space constraints or color figure charges

- Immediate publication on acceptance

- Inclusion in PubMed, CAS, Scopus and Google Scholar

- Research which is freely available for redistribution

Submit your manuscript at www.biomedcentral.com/submit
C) BioMed Central 